\title{
Reflectarray Antennas for Dual Polarization and Broadband Telecom Satellite Applications
}

\author{
Rafael Florencio, José A. Encinar, \\ Giovanni Toso
}

\begin{abstract}
A reflectarray antenna with improved performance is proposed to operate in dual-polarization and transmit-receive frequencies in $\mathrm{Ku}$-band for broadcast satellite applications. The reflectarray element contains two orthogonal sets of four coplanar parallel dipoles printed on two surfaces, each set combining lateral and broadside coupling. A $40-\mathrm{cm}$ prototype has been designed, manufactured and tested. The lengths of the coupled dipoles in the reflectarray cells have been optimized to produce a collimated beam in dual polarization in the transmit and receive bands. The measured radiation patterns confirm the high performance of the antenna in terms of bandwidth $(27 \%)$, low losses and low levels of cross polarization. Some preliminary simulations at $11.95 \mathrm{GHz}$ for a $1.2-\mathrm{m}$ antenna with South American coverage are presented to show the potential of the proposed antenna for spaceborne antennas in Ku-band.
\end{abstract}

Index Terms-Reflectarrays, broadband antennas, dipole arrays, cross polarization, satellite antennas.

\section{INTRODUCTION}

THE antenna requirements for broadcast and telecommunication satellites are becoming very challenging, including well defined contoured beams, dual-polarization, transmit-receive $(\mathrm{Tx}-\mathrm{Rx})$ operation, and high co-polar isolation. Conventional shaped reflectors can provide these stringent requirements at the cost of a significant mass, volume and mechanical complexity, since either Gregorian or dual-gridded configurations are required to achieve low cross-polarization requirements [1] (Ch. 12).

A reflectarray antenna consists of a planar array of reflective elements illuminated by a feed (see Fig. 1), where the phase-shift introduced by each element is pre-adjusted to appropriately shape the beam [2]. In most practical cases, the phase adjustment is implemented by printed patches with

\author{
Rafael R. Boix
}

Vicente Losada, attached [3] or aperture-coupled delay lines[4]-[6], or by using variable size printed elements in one [7]-[11] or several layers [12]-[13].

Printed reflectarrays show several advantages when compared to conventional reflector antennas for space applications [14], [1] (Ch. 10). First, contoured beams can be easily generated by using a flat reflectarray made of printed patches properly optimized [15]-[16]. Second, the mechanical design is simplified because it is reduced to a single flat sandwich. Third, the manufacturing time and cost are reduced due to the elimination of the custom molds required for conventional shaped reflectors. Fourth, reflectarrays offer an independent phase control on each polarization, which can be used to produce a different beam in each polarization [16][17]. Finally, reflectarrays could provide in-flight reconfigurability of the coverage [18], provided that electronically controllable phase-shifters are included at element or sub-array level [19].

Although the narrow bandwidth is a severe limitation in reflectarray antennas [20], a significant effort has been done in the last few years to improve it. Different broadband reflectarray elements have been proposed, including stacked patches of variable size [12]-[13], aperture coupled elements [5], modified Malta crosses [8] and multi resonant elements on

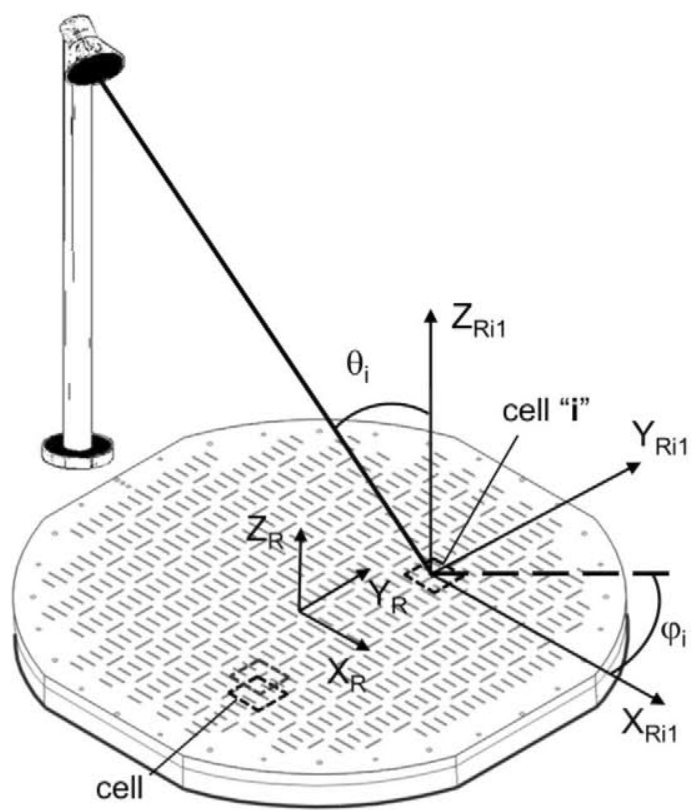

ig. 1. Drawing of reflectarray antenna, including coordinate systems for eflectarray and for cell " $i$ ". 
a single substrate layer [9]-[11]. In all these configurations, the element bandwidth is improved by adding more degrees of freedom to control the phase in the reflectarray cell, and by adjusting the associated geometrical parameters [21].

The use of broadband reflectarray elements is a necessary prerequisite but it is not sufficient to design large reflectarrays, such as those that are required in space applications. In this latter case the different path lengths produce several steps of $360^{\circ}$ in the phase on the reflectarray surface, as discussed in [22] and [2] (Ch. 5). This issue is even more significant for contoured-beam antennas as demonstrated in [23].

Different techniques have been proposed to improve the bandwidth in large reflectarrays, such as the use of delay lines providing a phase range of several times $360^{\circ}$ [24], the use of facetted configurations approximating a paraboloid [25]-[26], or the use of reflectarray elements that are directly printed on a parabolic surface [27]-[28]. Although the faceted or parabolic reflectarrays considerably improve the bandwidth, the cross-polarization is penalized by the panels' inclination or by the offset parabolic surface [28]. Additional optimizations would be required to design an antenna for $\mathrm{Tx}-\mathrm{Rx}$ frequencies and to reduce the cross-polarization.

Another technique to improve the bandwidth in large reflectarrays consists of using multi-resonant elements, such as three stacked rectangular patches, and optimizing the resonant dimensions to compensate for the spatial phase delay at several frequencies [29]. This latter reference shows that three degrees of freedom (associated to 3 stacked patches) are necessary in the optimization process to design a $1-\mathrm{m}$ antenna at Ku-band for a $10 \%$ bandwidth. The optimization at several frequencies has been applied to design reflectarray antennas based on multi-resonant elements, either on a single layer [9] or in a stacked configuration [16], up to a $15 \%$ bandwidth in Ku-band [30]. Recently, a 1.2-meter reflectarray has been designed for a DBS mission operating in Tx (11.7-12.2 GHz) and $\mathrm{Rx}(13.75-14.25 \mathrm{GHz})$ frequency bands to provide a South American coverage in dual-linear polarization [31]. The results obtained for this prototype show that a reflectarray can be designed to fulfill the typical requirements of Tx-Rx DBS antennas for the co-polar radiation patterns. However, the required level of isolation between orthogonal polarizations in contoured beam DBS antennas (typically $30 \mathrm{~dB}$ ) is hard to be achieved with the configuration of stacked patches, particularly for a wide contoured beam, as shown in [16].

The cross-polarization for contoured-beam reflectarray antennas can be reduced by a direct optimization technique as proposed in [32], where one geometrical parameter is optimized to fulfill the requirements of co- and cross-polar radiation patterns at several frequencies. This can be achieved either with a single layer of square patches [32], or with concentric squared loops and patches [33]. The use of this technique has made it possible to achieve a cross-polar discrimination (XPD) of $27 \mathrm{~dB}$ in a $20 \%$ bandwidth. However, a reflectarray element with more degrees of freedom in the geometry is necessary to simultaneously meet the requirements of coverage and cross-polarization in both $\mathrm{Tx}$ and Rx frequency bands.
The cross-polarization produced by the reflectarray elements can be reduced by using independent dipoles of variable size for each polarization [34]-[36]. The broadband reflectarray elements based on three coplanar parallel dipoles [10] can be used for dual polarization by adding a second arrangement of three parallel dipoles, perpendicular to the first set of dipoles, as proposed in [36]. The dimensions of the parallel dipoles associated to each polarization can be independently optimized for bandwidth improvement, as in the case of reflectarrays based on stacked patches [29]. However, each pair of lateral parallel dipoles should be kept identical to preserve symmetry in the reflectarray element, which ensures a low cross-polarization. As a result, only two degrees of freedom (length of central and lateral dipoles) for each polarization can be used in the optimizations, which are not enough to design a 1-m antenna in Ku-band [29].

In this paper we investigate several configurations of reflectarray elements based on sets of parallel dipoles that provide enough degrees of freedom to design high performance reflectarray antennas operating in dualpolarization and $\mathrm{Tx}-\mathrm{Rx}$ frequencies in Ku-band for telecommunications and broadcast satellite applications. A new reflectarray element is proposed that provides three degrees of freedom for each polarization. This is achieved by combining lateral and broadside coupled dipoles in only two levels of metallization, which simplifies the manufacturing process and reduces the antenna cost. The proposed reflectarray element shows a very good performance in Tx and $\mathrm{Rx}$ frequency bands with very low levels of cross-polarization. A $40-\mathrm{cm}$ reflectarray has been designed, manufactured and tested. The measured radiation patterns confirm the high performance of the antenna in terms of bandwidth, low losses and low cross-polarization. Finally, preliminary simulation results are presented for a 1.2-m reflectarray antenna, which is designed to provide South American coverage at $11.95 \mathrm{GHz}$. Although all the results are presented for Ku-band, the proposed configuration can be used to provide around $25 \%$ bandwidth at other frequencies, by scaling the geometry and optimizing the dipole dimensions.

\section{REFLECTARRAY ELEMENT FOR BROADBAND AND LOW} CROSS-POLARIZATION

Several reflectarray cells based on sets of parallel dipoles for each linear polarization, which include additional laterally or broadside coupled parallel dipoles, are studied in order to provide more degrees of freedom (i.e. more resonances) that will be adjusted during the optimization process. The sets of dipoles are perpendicularly oriented to each other. It has been checked by e.m. simulations that the cross-polarization introduced by this type of reflectarray elements is lower when the parallel dipoles are symmetric in the periodic cell, at the cost of reducing the degrees of freedom (independent dipole lengths). Four coplanar parallel dipoles have not been chosen because they only provide 2 optimization variables for each polarization in a symmetric configuration, as it happens in the case studied in [21] where three parallel dipoles were used. In the following, an odd number of laterally coupled dipoles will be assumed, the lateral dipoles being symmetric with respect 
to the central dipole, in order to keep low levels of crosspolarization.

In the study of the reflectarray, the cells will be assumed to be placed in a periodic environment as typically done in the analysis and design of a reflectarray antennas [2], [7]. The reflectarray cell is characterized by the reflection matrix $(\mathbf{R})$, which accounts for the co- and cross-polar reflection coefficients for both polarizations. The $\mathbf{R}$ matrix relates the cartesian complex components of the reflected and incident tangential electric field on the reflectarray cell in a periodic environment as shown below:

$$
\left(\begin{array}{l}
E_{x}^{r e f} \\
E_{y}^{r e f}
\end{array}\right)=\left(\begin{array}{ll}
R_{x x} & R_{y x} \\
R_{x y} & R_{y y}
\end{array}\right) \cdot\left(\begin{array}{l}
E_{x}^{i n c} \\
E_{y}^{i n c}
\end{array}\right)
$$

The $\mathbf{R}$ matrix for the reflectarray elements considered in this section are computed with a home-made software based on the Method of Moments in the Spectral Domain (MoM-SD) [37] with multilayered Green's functions [38], which has been validated by comparing their results with those obtained with the commercial software $\mathrm{CST}^{\mathbb{B}}[39]$.

The following broadband reflectarray elements are designed by properly adjusting the different geometrical parameters. First, the period is fixed to allow the maximum required dipole lengths and to avoid the appearance of grating lobes [2] (p. 84). For each element, a parametric study has been carried out by varying the substrate thickness, the dipole separations and the relative dipole lengths. Commercially available materials have been selected, and the thickness and permittivity have been chosen to avoid sharp resonances and to provide an appropriate coupling for the stacked dipoles. Once the period and lay-up have been defined, the relative lengths of the parallel dipoles are adjusted for each polarization to properly place the resonances (minimum of reflection coefficient) in order to provide a smooth and linear phase response in the prescribed range of frequencies, as in the case of three parallel dipoles [40]. The elements are designed to operate in dual polarization in two frequency bands centered at $11.95 \mathrm{GHz}$ for Tx and $14.00 \mathrm{GHz}$ for $\mathrm{Rx}$.

\section{A. Two-Layer Reflectarray Cell with Five Dipoles for Each Polarization.}

The first cell includes five parallel dipoles for each polarization, as shown in Fig. 2(a). Each set of parallel dipoles is printed on the opposite sides of a thin dielectric layer $\left(\mathrm{h}_{\mathrm{B}}=0.127 \mathrm{~mm}\right.$ of thickness) separated from a ground plane by a second dielectric layer of thickness $h_{\mathrm{A}}$. The novelty of this element is to provide 3 independent dipole lengths for each polarization in only two layers. To reduce the RF losses, a commercial low-loss material is used for the two dielectric layers, Diclad ${ }^{\mathrm{TM}} 880$, with permittivity $\varepsilon_{\mathrm{T}}=$ 2.17 and loss tangent $\tan \delta=0.0009$. Honeycomb can also be used instead as a separator to reduce the mass in space antennas. In accordance with the procedure described above, the following dimensions (see Fig. 2(a)) have been chosen: period $\mathrm{P}_{\mathrm{X}}=\mathrm{P}_{\mathrm{Y}}=13 \mathrm{~mm}$, separator thickness $\mathrm{h}_{\mathrm{A}}=3.175 \mathrm{~mm}$, equal separation between dipoles $\mathrm{S}_{\mathrm{B} 2}=\mathrm{S}_{\mathrm{A} 2}=2.1 \mathrm{~mm}, \mathrm{~S}_{\mathrm{B} 1}=$ $\mathrm{S}_{\mathrm{A} 1}=4.7 \mathrm{~mm}$, dipole widths $\mathrm{w}=0.5 \mathrm{~mm}$, relative sizes of lateral dipoles $l_{\mathrm{B} 1}=0.6 l_{\mathrm{B} 3}, l_{\mathrm{B} 2}=0.45 l_{\mathrm{B} 3}, l_{\mathrm{A} 1}=0.6 l_{\mathrm{A} 3}, l_{\mathrm{A} 2}=$
$0.45 l_{\mathrm{A} 3}, l_{\mathrm{B} 3}=l_{\mathrm{A} 3}=l$. For these geometrical parameters, the magnitude and phase of co-polar reflection coefficients at normal incidence in $\mathrm{Tx}(11.3 \mathrm{GHz}$ and $12.6 \mathrm{GHz})$ and $\mathrm{Rx}$ $(14.00 \mathrm{GHz})$ frequencies are shown in Fig. 2(b) and (c) as a function of the dipole lengths for X-polarization. A similar response is obtained for Y-polarization. In spite of the presence of 5 dipoles, the results do not show a significant improvement with respect to those obtained for three dipoles [21]. In fact, only two resonances and a range of phase-shift around $400^{\circ}$ is obtained for the lower frequency. It has been checked that a larger phase range (two cycles of $360^{\circ}$ ), produced by the effect of three resonances, is required to design a reflectarray in $\mathrm{Tx}$ and $\mathrm{Rx}$ bands with low levels of cross-polarization. One way to have more resonances in the cell is to combine the laterally-coupled parallel dipoles with dipoles in stacked configuration, as shown in the next subsections.

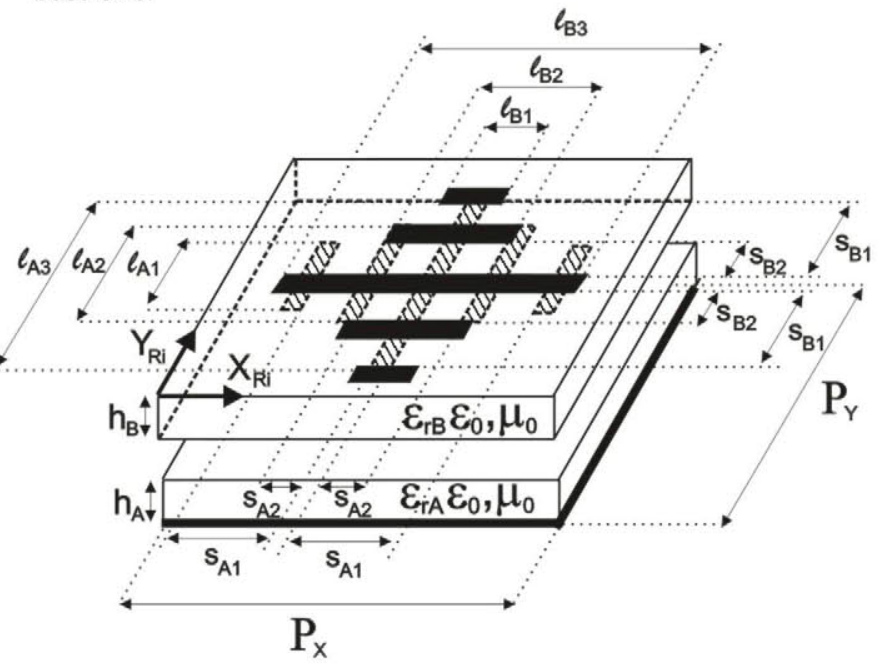

(a)

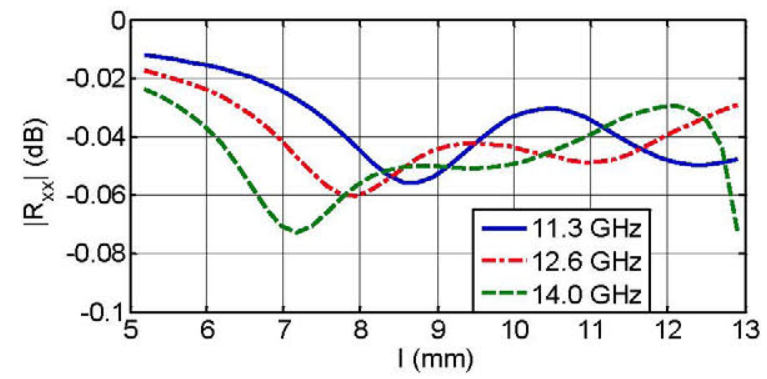

(b)

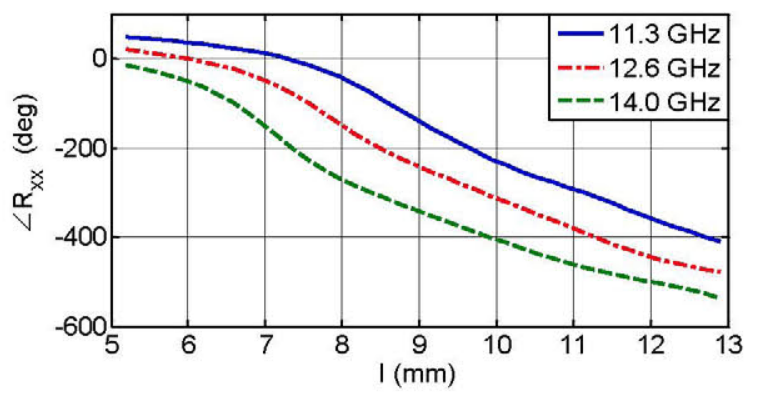

(c)

Fig. 2. Reflectarray element based on five parallel dipoles for each polarization. (a) Exploded view. (b) Magnitude and (c) phase of co-polar reflection coefficients for X-polarization at normal incidence vs. the length of central dipole at different frequencies. 
B. Four-Layer Reflectarray Cell with Four Dipoles for Each Polarization.

One solution to achieve a reflectarray cell with more resonances is to stack two layers of dielectric substrates with parallel dipoles printed on each side (four metallization levels). To provide three resonances, only one additional stacked dipole has to be added to the three symmetric parallel dipoles for each polarization, as shown in Fig. 3(a). The proposed cell consists of 4 dielectric layers (A, B, C and D) with printed dipoles. The phase-shift for Y-polarization is controlled at different frequencies by adjusting the lengths of the three dipoles printed on top of layer B and the one on top of layer C, see Fig. 3(a). In a similar way, the phase for X-

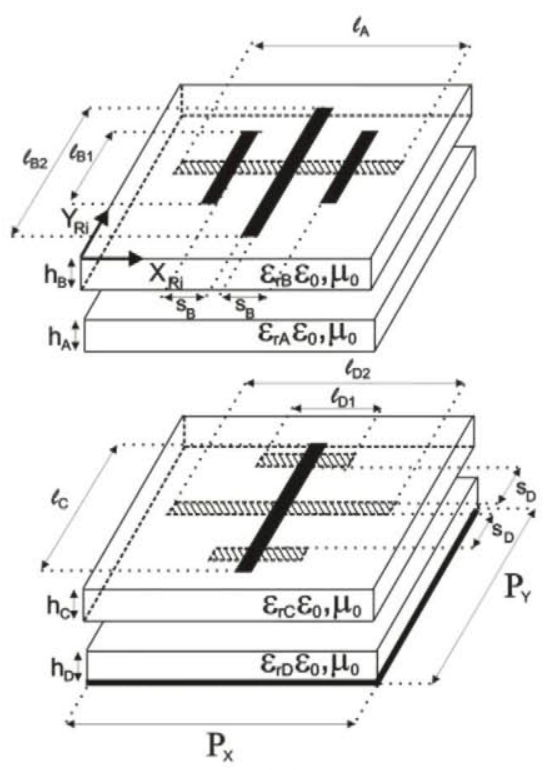

(a)

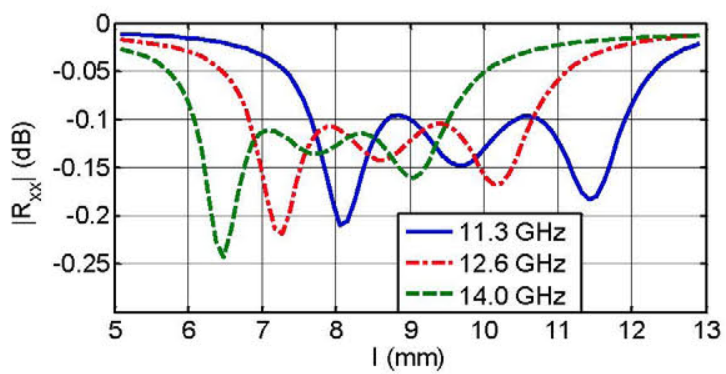

(b)

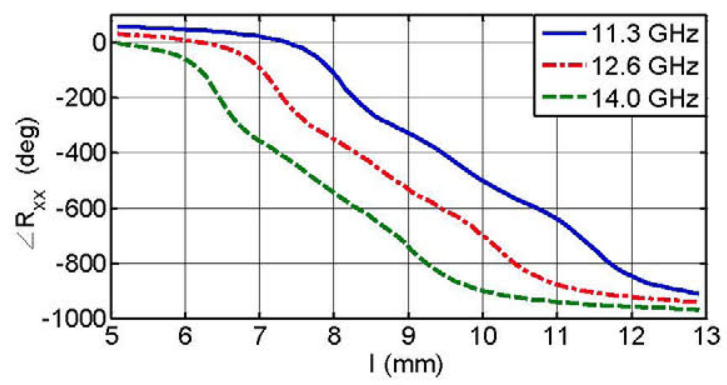

(c)

Fig. 3. Reflectarray element based on four parallel dipoles for each polarization in four levels. (a) Exploded view. (b) Magnitude and (c) phase of co-polar reflection coefficients for X-polarization at normal incidence vs. the length of central dipole at different frequencies. polarization is controlled by the lengths of the four dipoles printed in the X-direction. The novelty of this element is that it combines lateral and stacked coupled dipoles to increase the phase range and number of resonances, while keeping an independent phase control for each polarization.

To simplify the manufacturing process, the dipoles are printed on the opposite sides of the thin dielectric layers B and $\mathrm{C}\left(\mathrm{h}_{\mathrm{B}}=\mathrm{h}_{\mathrm{C}}=0.127 \mathrm{~mm}\right.$ of thickness) separated by a thicker dielectric layer $\mathrm{A}$ and placed above a grounded dielectric $\mathrm{D}$. The same Diclad material has been used with thickness $h_{B}=$ $\mathrm{h}_{\mathrm{C}}=0.127 \mathrm{~mm}, \mathrm{~h}_{\mathrm{A}}=\mathrm{h}_{\mathrm{D}}=1.5 \mathrm{~mm}$, but 2-mm honeycomb could be used in layers A and D for space antennas. After the adjustment of the dimensions, the following values have been chosen to provide a smooth phase response in $\mathrm{Tx}$ and $\mathrm{Rx}$ frequencies: period $\mathrm{P}_{\mathrm{X}}=\mathrm{P}_{\mathrm{Y}}=13 \mathrm{~mm}, \mathrm{~S}_{\mathrm{B}}=\mathrm{S}_{\mathrm{D}}=4.5 \mathrm{~mm}, l_{\mathrm{B} 2}=$ $0.93 l_{\mathrm{C}}, l_{\mathrm{B} 1}=0.6 l_{\mathrm{C}}, l_{\mathrm{A}}=0.73 l_{\mathrm{D} 2}, l_{\mathrm{D} 1}=0.8 l_{\mathrm{D} 2}$. The magnitude and phase at normal incidence in $\mathrm{Tx}$ and $\mathrm{Rx}$ frequencies as a function of the dipole lengths $l_{\mathrm{C}}=l_{\mathrm{D} 2}=l$ for X-polarization are shown in Fig. 3(b) and (c), while a similar response is obtained for Y-polarization. The losses produced by the dielectric materials are directly the magnitude in $\mathrm{dB}$ of the reflection coefficient shown in Fig. 3(b) but with opposite sign. The losses are lower than $0.25 \mathrm{~dB}$, with an average value around $0.12 \mathrm{~dB}$. The main improvement of this element is that it provides a much larger phase range (more than two cycles of $360^{\circ}$ ) and more degrees of freedom for the optimizations [29].

The amplitude and phase responses for co- and cross-polar

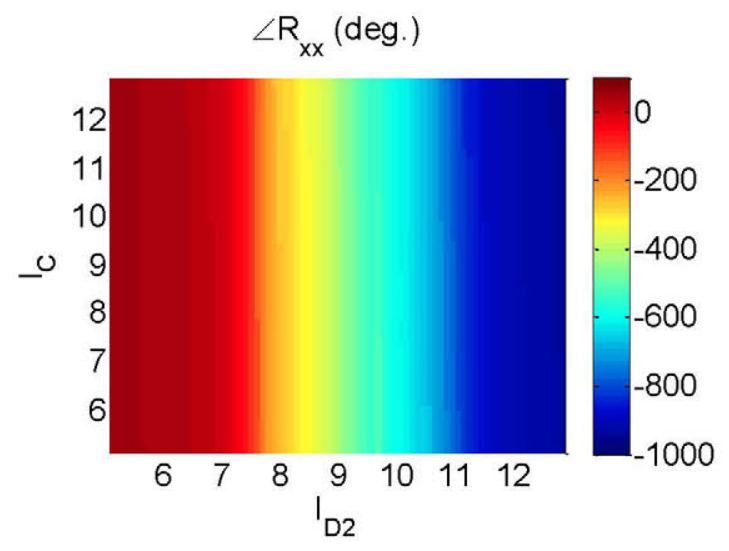

(a)

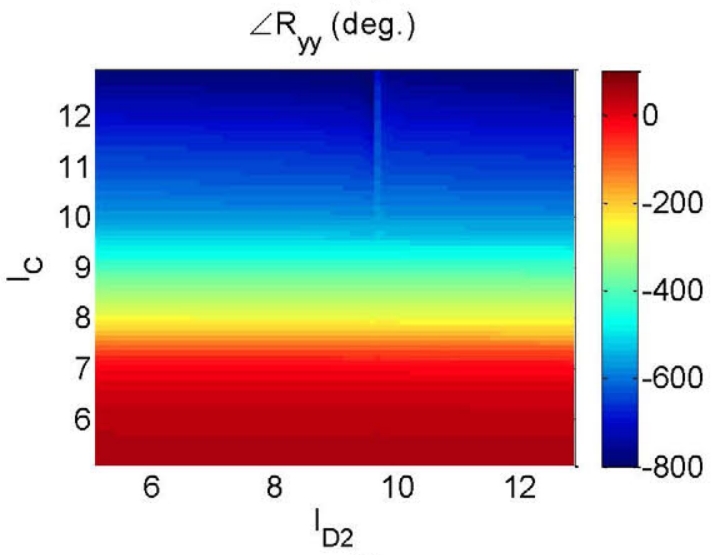

(b)

Fig. 4. Phase response vs. the sizes of dipoles for $\mathrm{X}$-polarization (a) and $\mathrm{Y}$ polarization (b) at oblique incidence $\left(\theta_{i}=\phi_{t}=30^{\circ}\right)$ for the 4-layer reflectarray cell. Results are presented at $11.95 \mathrm{GHz}$ 
components have been studied for oblique incidence $\left(\theta_{i}=\phi_{i}=\right.$ $30^{\circ}$ ) in $\mathrm{Tx}$ and $\mathrm{Rx}$ frequency bands when the length of dipoles for both polarizations are varied. The phasing performance for both polarizations is shown in Fig. 4 at $11.95 \mathrm{GHz}$. These figures clearly show that the phase control is completely independent for both polarizations, even at oblique incidence, within a range of phase-shift larger than $800^{\circ}$. Note that independent phase control makes it possible a different beam forming in each polarization as demonstrated in [16] and [17], which cannot be achieved with other multiple ring elements as those reported in [9] and [13]. As an additional advantage of this element, the magnitude of the cross-polar components of the $\mathbf{R}$ matrix is very low for both polarizations and for practically all combinations of dipole lengths as shown in Fig. 5 . The cross-polarization only increases in the area close to the dimensions $l_{\mathrm{D} 2}=9.5 \mathrm{~mm}, l_{\mathrm{C}}=12.0 \mathrm{~mm}$, due to a spurious resonance. We have observed that this type of resonances also appear in other reflectarray elements, as in the case of three parallel dipoles and stacked patches [21], for a certain combination of dimensions and angle of incidence (out of the principal planes). For these combinations of dipole lengths and angle of incidence, which should be avoided in a practical design, some energy is transferred from co-polar to cross-polar components. In general, we have observed that these spurious resonances are reduced for a smaller period, e.g., when the period is reduced to $12 \mathrm{~mm}$ in this case. This reflectarray cell could be used to design reflectarray antennas with low cross-
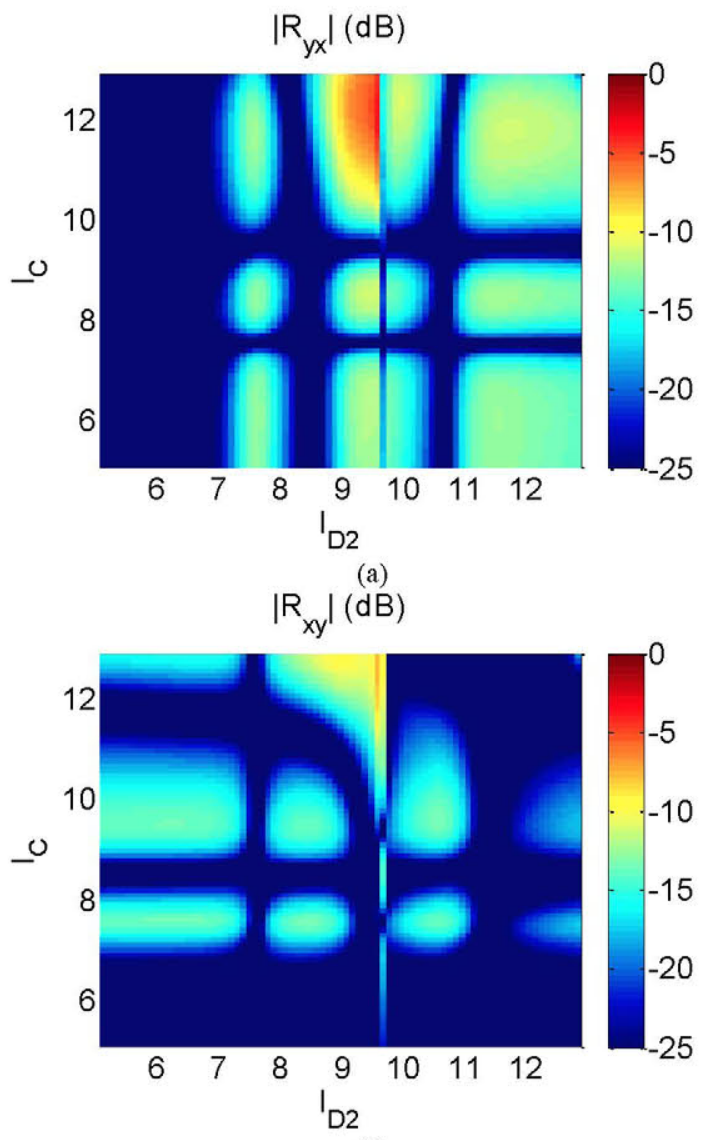

(b)

Fig. 5. Magnitude of cross-polar components vs. the sizes of dipoles for $X-$ polarization (a) and Y-polarization (b) at oblique incidence $\left(\theta_{i}=\phi_{t}=30^{\circ}\right)$ for the 4-layer reflectarray cell. Results are presented at $11.95 \mathrm{GHz}$. polarization to operate in transmit $(11.3-12.6 \mathrm{GHz})$ and receive $(13.75-14.25 \mathrm{GHz})$ frequencies for $\mathrm{Ku}$-band space antennas. Even though this configuration requires two dielectric sheets with dipoles printed on both sides and two separator layers instead of the three array layers and three honeycomb separators used in previous demonstrators for space DBS antennas [16], [31], the four levels of metallization involved would still penalize the complexity and cost of manufacturing.

\section{Two-Layer Reflectarray Cell with Four Dipoles for Each Polarization.}

The number of array layers in Fig. 3(a) can be reduced from 4 to 2 by shifting half-a-period the set of dipoles associated to one polarization, and by printing on the same surface three parallel dipoles for one polarization and one dipole for the orthogonal polarization. The resulting element, shown in Fig. 6 , consists of two sets of dipoles displaced one from each other. Each set consists of three coplanar parallel dipoles
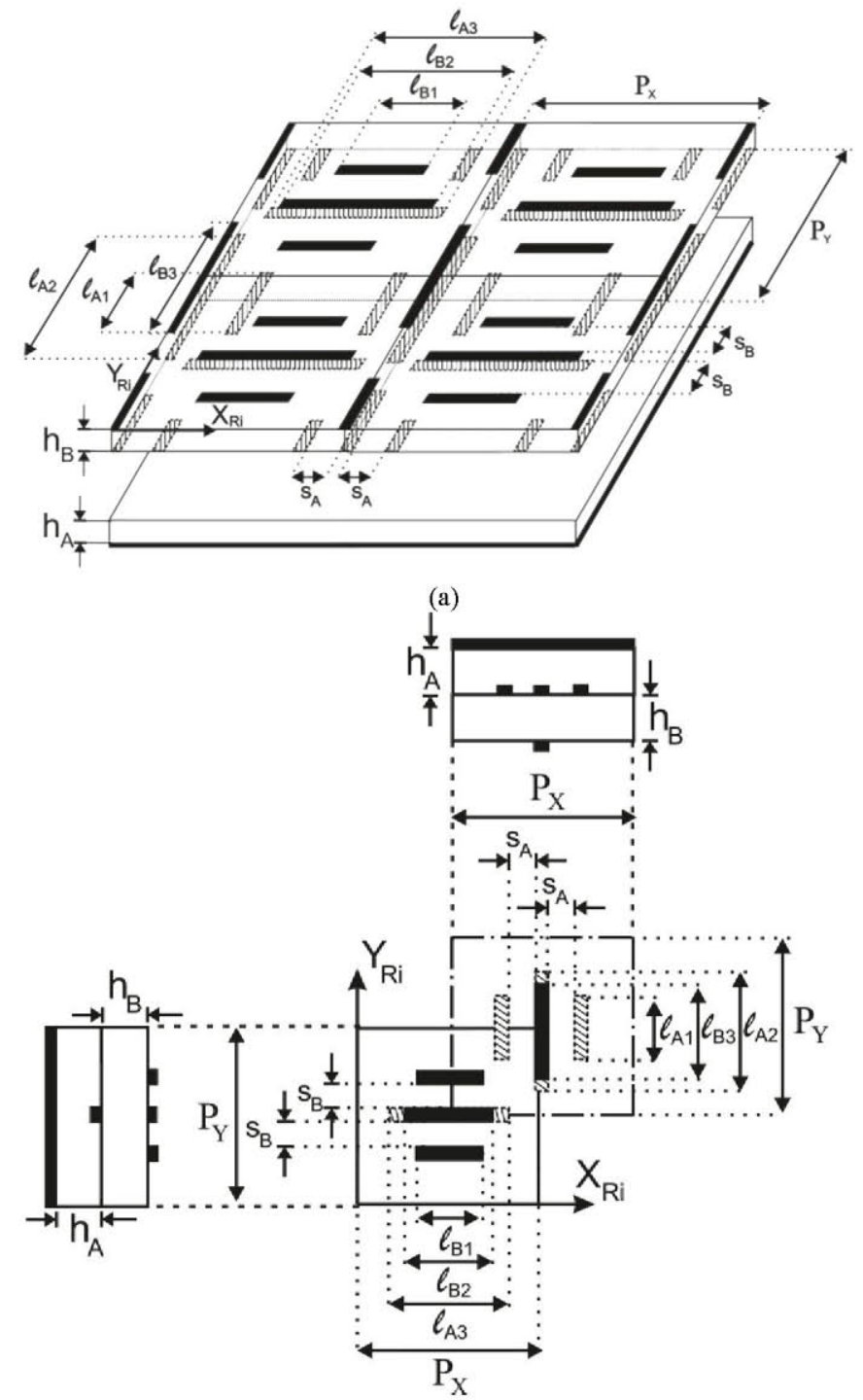

(b)

Fig. 6. Reflectarray element made of four lateral and stacked dipoles for each polarization in two levels of metallization. (a) Exploded view of four periodic cells. (b) Top and side views of a phasing cell for X- and Ypolarization. 
printed on a dielectric layer and a fourth parallel dipole, which is stacked with the central one and is printed on the opposite side of the same dielectric sheet (B). In the case of Xpolarization, the three horizontal parallel dipoles are printed on the top surface and the fourth dipole on the bottom surface, while the placement of the dipoles for Y-polarization is the other way round (see Fig. 6). The following parameters have been chosen to provide a smooth phase response in $\mathrm{Tx}$ and $\mathrm{Rx}$ frequencies: period $\mathrm{P}_{\mathrm{X}}=\mathrm{P}_{\mathrm{Y}}=12 \mathrm{~mm}$; dielectric layers parameters $\varepsilon_{\mathrm{rA}}=2.55, \tan \delta_{A}=0.0009, \mathrm{~h}_{\mathrm{A}}=2.363 \mathrm{~mm}, \varepsilon_{\mathrm{rB}}=$ $2.17, \tan \delta_{B}=0.0009, \mathrm{~h}_{\mathrm{B}}=1.5 \mathrm{~mm}$; equal separation between laterally coupled dipoles $\mathrm{s}_{\mathrm{B}}=\mathrm{s}_{\mathrm{A}}=3.5 \mathrm{~mm}$; dipole width $\mathrm{w}=$ $0.5 \mathrm{~mm}$; and relative sizes of lateral dipoles $l_{\mathrm{A} 1}=0.58 l_{\mathrm{A} 2}, l_{\mathrm{B} 3}=$ $0.95 l_{\mathrm{A} 2}, l_{\mathrm{B} 2}=0.93 l_{\mathrm{A} 3}, l_{\mathrm{B} 1}=0.63 l_{\mathrm{A} 3}$. For this periodic cell, the magnitude and phase in $\mathrm{Tx}$ and $\mathrm{Rx}$ frequencies for $\mathrm{X}$ polarization are shown in Fig. 7 as a function of the dipole lengths $l_{\mathrm{A} 3}=l_{\mathrm{A} 2}=l$. In this case the angle of incidence has been chosen as $\theta_{i}=16.9^{\circ}, \phi_{i}=0^{\circ}$, which corresponds to the incidence angle on the center of the reflectarray designed in the next section. For this angle of incidence, the amplitude and phase curves are very similar to those obtained for normal incidence, which are also included in Fig. 7. A linear phase response in a large phase range (larger than two cycles of $360^{\circ}$ at $12.6 \mathrm{GHz}$ ) is achieved by adjusting the relative lengths of the dipoles. These results are similar to those shown in the previous subsection for an element with four array layers. The expected average losses are lower than $0.1 \mathrm{~dB}$. As it happens with the element studied in the previous subsection, the phase

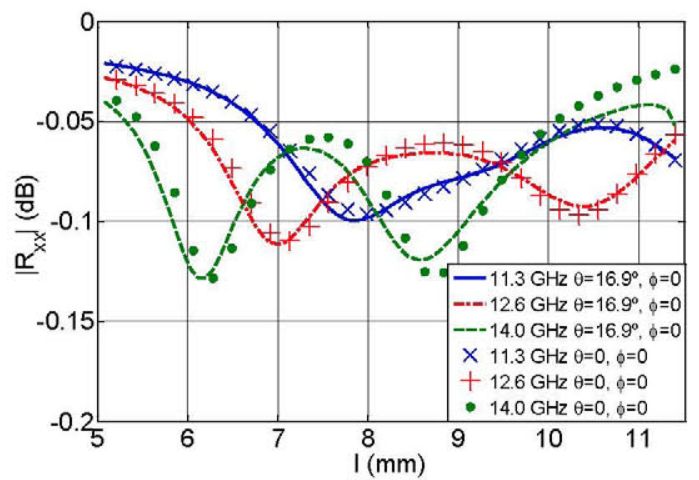

(b)

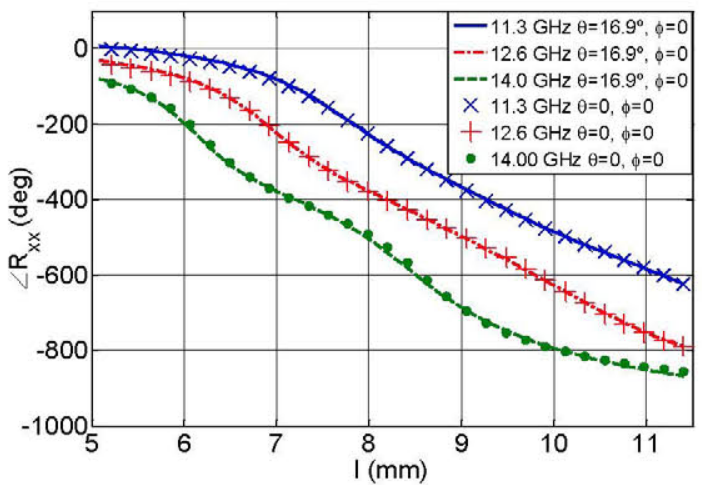

(c)

Fig. 7. Magnitude (a) and phase (b) of co-polar reflection coefficients for Xpolarization at both oblique incidence $\left(\theta=16.9^{\circ} \phi=0^{\circ}\right)$ and normal incidence $\left(\theta=\phi=0^{\circ}\right)$ vs. the length of central dipole for the 2-layer reflectarray element with $4+4$ dipoles. Results are presented at different frequencies. control is completely independent for both polarizations within a phase range close to $700^{\circ}$ as shown in Fig. 8, and the cross-polar amplitudes of the R matrix are very low for both polarizations ( $<-10 \mathrm{~dB}$ for X-pol. and $<-12 \mathrm{~dB}$ for Y-pol.) as shown in Fig. 9. Note that the relative lengths of the dipoles in one polarization with respect to the other can be adjusted to reduce the cross-polarization, which can be implemented by adding a constant to the phase distribution in one polarization.

In conclusion, the three reflectarray elements shown in this

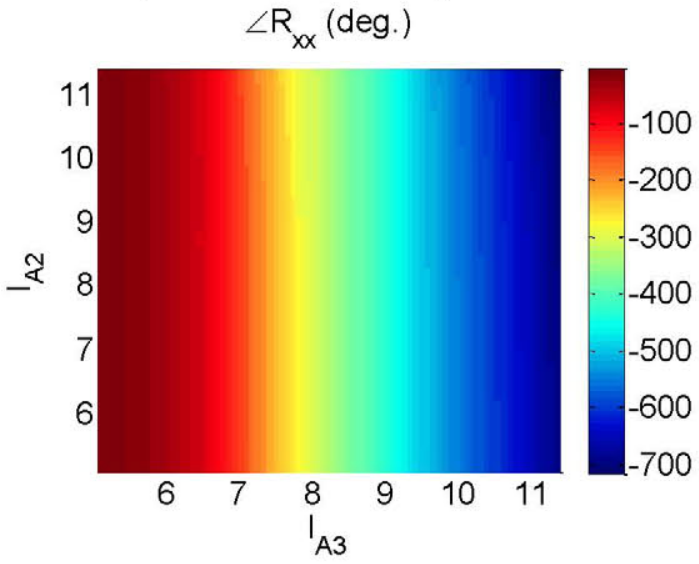

(a)

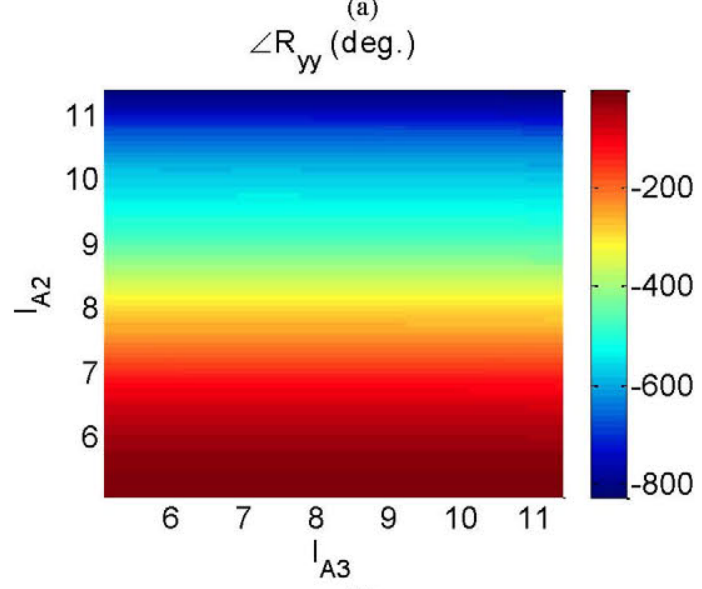

(b)

Fig. 8. Phase response vs. the sizes of dipoles for X-polarization (a) and Ypolarization (b) at oblique incidence $\left(\theta_{i}=\phi_{t}=30^{\circ}\right)$ for the 2-layer reflectarray element with $4+4$ dipoles. Results are presented at $11.95 \mathrm{GHz}$.

section exhibit a linear phase response and low losses $(<0.2 \mathrm{~dB})$ for dual polarization, covering $\mathrm{Tx}$ and $\mathrm{Rx}$ frequencies in Ku-band. They also permit an independent phase control for each polarization. The reflectarray element presented in this subsection and the previous four-layer element both provide low cross-polarization and a linear phase response in a large phase range $\left(>2 \times 360^{\circ}\right)$, which is not achievable with the element based on five parallel coplanar dipoles. These two elements present sufficient degrees of freedom (three independent dipole-lengths for each polarization) to be used in optimization procedures for the design of reflectarray antennas with low cross-polarization in transmit and receive frequencies for Ku-band. The main advantage of the latter reflectarray element with respect to the former is a reduction of mass and a simplification in manufacturing, since it only requires two layers with printed dipoles. 


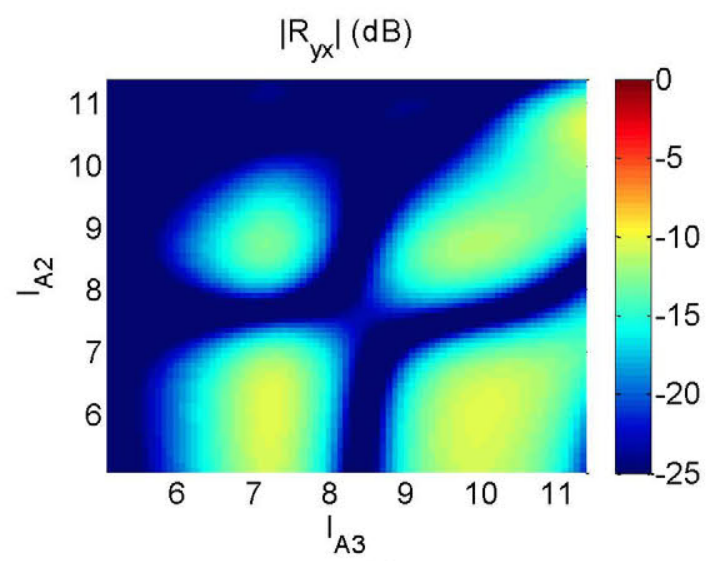

(a)

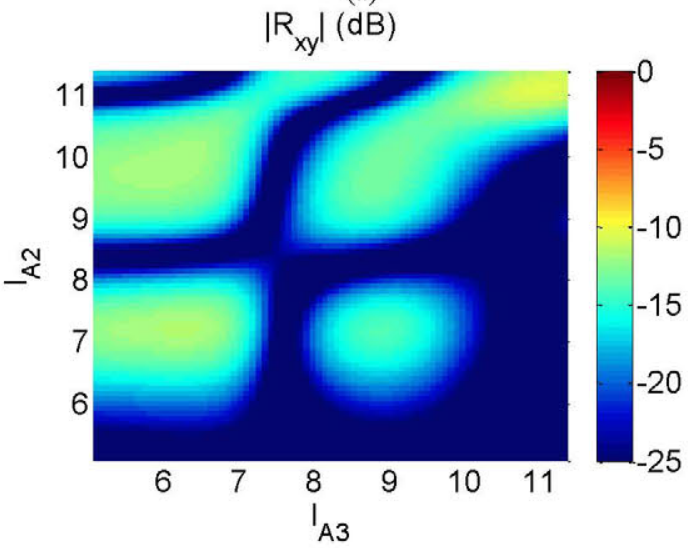

(b)

Fig. 9. Magnitude of cross-polar components vs. the sizes of dipoles for Xpolarization (a) and Y-polarization (b) at oblique incidence $\left(\theta_{i}=\phi_{i}=30^{\circ}\right)$ for the 2-layer reflectarray element with $4+4$ dipoles. Results are presented at $11.95 \mathrm{GHz}$.

\section{DESIGN OF REFLECTARRAY PROTOTYPE}

The two-layer reflectarray element characterized in subsection II.C has been used to design a $40-\mathrm{cm}$ reflectarray antenna that produces a focused beam in the direction $\theta_{b}=$ $16.9^{\circ}$ and $\varphi_{b}=0^{\circ}$ for the frequency bands $11.3-12.6 \mathrm{GHz}(\mathrm{Tx})$ and $13.75-14.25 \mathrm{GHz}(\mathrm{Rx})$ in both linear polarizations. The reflectarray can be designed for a higher scan angle, the maximum scan angle being limited by the appearance of grating lobes at the highest frequency, which is $48^{\circ}$ for a period of $12 \mathrm{~mm}$. The angle of the radiated beam and the incident angle from the feed to the reflectarray center are chosen identical to prevent beam squint [41] . This angle $\left(16.9^{\circ}\right)$ has been chosen small to minimize the crosspolarization produced by the printed dipoles, and large enough to ensure no blockage from the feed.

The reflectarray is circular with the elements arranged in a $33 \times 33$ grid for X-polarization and in a $32 \times 32$ grid for $\mathrm{Y}$ polarization. This is because some phasing cells for Ypolarization on the edge have been eliminated to maintain the antenna symmetric with respect to the coordinate plane $X_{R} Z_{R}$. The reflectarray is illuminated by a corrugated circular feedhorn with its phase center located at coordinates $X_{R}=-193$ $\mathrm{mm}, Y_{R}=0 \mathrm{~mm}$ and $Z_{R}=635 \mathrm{~mm}$ with respect to the center of the reflectarray. The radiation pattern of the corrugated horn has been modeled by the conventional $\cos ^{\mathrm{q}}(\theta)$ where ' $\mathrm{q}$ ' varies with frequency. The feed illuminates the edges of the reflectarray with a taper of $-10 \mathrm{~dB}$ for Tx-band and $-13 \mathrm{~dB}$ for Rx-band.

The phase-shift distribution required to focus the beam in one polarization has been shifted by $180^{\circ}$ with respect to the phase in the orthogonal polarization. This ensures that the dipoles controlling the X-polarization have dimensions that are different from those of the dipoles for the Y-polarization, which allows more room for the orthogonal dipoles printed on the same layer. In a first step, the reflectarray has been designed at a single frequency $(11.95 \mathrm{GHz})$ by keeping the relative lengths of dipoles defined in previous section, and by adjusting the lengths of each set of parallel dipoles to match the phase distribution required in each polarization. The lengths of the dipoles are found by a zero finding routine that iteratively calls the MoM-SD analysis tool. In this process, local periodicity is assumed and the angle of incidence in each element of the reflectarray is taken into account. In a second step, the three independent dimensions of the dipoles for each polarization $\left(l_{\mathrm{A} 3}, l_{\mathrm{B} 1}\right.$ and $l_{\mathrm{B} 2}$ for the X-polarization, and $l_{\mathrm{B} 3}, l_{\mathrm{A} 1}$ and $l_{\mathrm{A} 2}$ for the Y-polarization) are optimized in each element to simultaneously match the required phases at central and extreme frequencies in $\mathrm{Tx}(11.3-12.6 \mathrm{GHz})$ and $\mathrm{Rx}(13.75-$ $14.25 \mathrm{GHz}$ ), following a procedure parallel to that in [29] and [31]. Since the phasing performance is practically uncoupled in the two polarizations as shown in previous section, the optimization is independently run for each polarization.

Once the optimization is completed and all the dipole lengths are defined, the radiation patterns are computed from the tangential electric and magnetic fields reflected at each reflectarray element according to the First Principle of

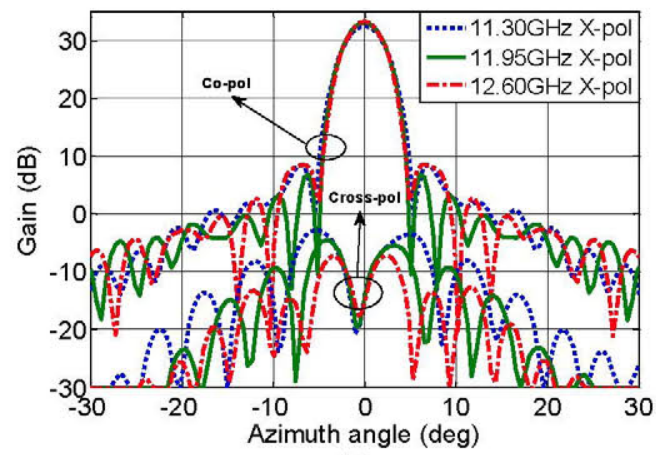

(a)

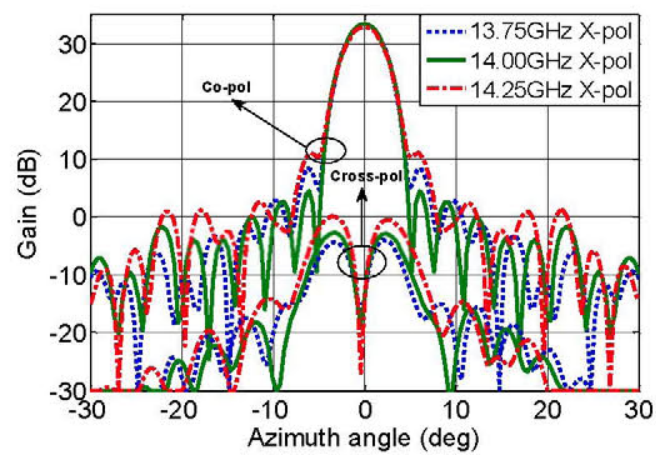

(b)

Fig. 10. Simulated radiation patterns in azimuth plane for X-polarization at $\mathrm{Tx}(\mathrm{a})$ and $\mathrm{Rx}$ (b) frequencies. 
Equivalence in electromagnetics [42]. The field components are computed by using the home-made MoM-SD tool under local periodicity assumption. The antenna gain is computed by taking into account the power radiated by the corrugated horn and the radiation patterns are computed with respect to the isotropic antenna (in dBi). Fig. 10 shows the simulated radiation patterns for $\mathrm{X}$-polarization at $\mathrm{Tx}$ and $\mathrm{Rx}$ frequencies in the azimuth plane (forming an angle $\theta_{b}=16.9^{\circ}$ with $Z_{\mathrm{R}}$ axis and perpendicular to $X_{R} Z_{R}$ plane). These patterns show low side lobes ( $23 \mathrm{~dB}$ below the maximum of co-polar radiation) and low cross polarization $(33 \mathrm{~dB}$ below the maximum of the co-polar beam) for all the frequencies in Tx and Rx. Similar radiation patterns are also obtained for Y-polarization. The radiation pattern in elevation plane $\left(X_{R} Z_{R}\right.$ in Fig. 1) also shows low levels of side lobes and cross-polarization below $13 \mathrm{dBi}$ because of the antenna symmetry.

This antenna is a limited-size demonstrator to evaluate the bandwidth and cross-polarization performance for potential applications in Ku-band spaceborne antennas, but the same antenna configuration can be used for the Earth stations or end-user terminals in Ku-band. In this case, Tx and $\mathrm{Rx}$ frequencies will be exchanged with respect to the definitions given in this paper.

\section{Manufacture AND Test of REFlectarRay PROTOTYPE}

The designed antenna prototype has been manufactured and tested. The two Diclad substrates, one with printed dipoles on both sides and the other backed by the ground plane, have been bonded by using two layers of thermoplastic bonding film (CuClad6250). Both substrates have been extended on the edges to have a frame without dipoles used to fix the antenna to an aluminum plate with nylon screws. The shape of the manufactured reflectarray panel results from the intersection of a circle of diameter $454 \mathrm{~mm}$ and a square of side $430 \mathrm{~mm}$, as can be seen in Figs. 1 and 11. A supporting structure made of aluminum cross-section profiles has been manufactured to fix the feed and reflectarray in the correct position.

The breadboard has been measured in a spherical near field antenna range in an anechoic chamber at the University of Seville. Figure 11 shows a picture of the reflectarray breadboard in the anechoic chamber. The measurements have been carried out for both polarizations $(\mathrm{X}$ and $\mathrm{Y})$ in the angular range $\left(-90^{\circ}<\theta<90^{\circ},-90^{\circ}<\varphi<90^{\circ}\right)$. The numerical and experimental results obtained for the 3D copolar and cross-polar components of the radiated field at 11.95

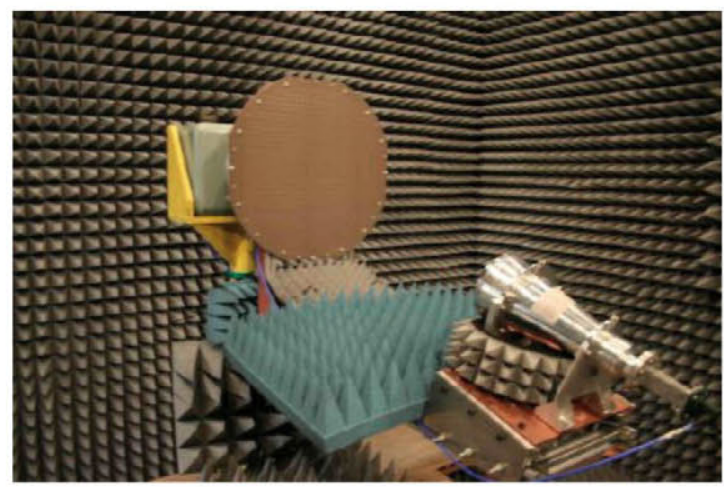

Fig. 11. Reflectarray prototype in the anechoic chamber.

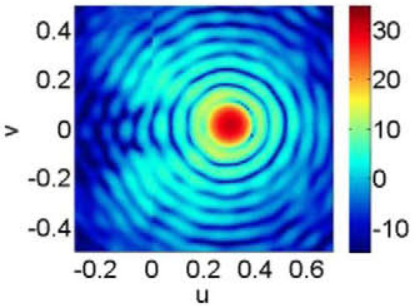

(a)

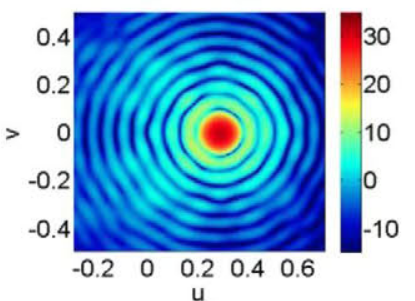

(c)

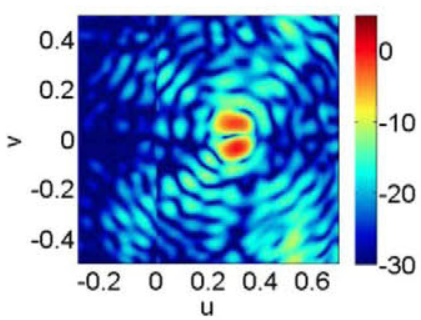

(b)

(d)

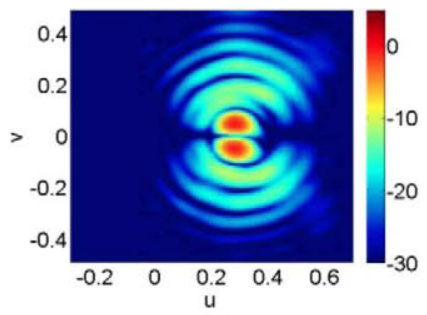

Fig. 12. 3D co-polar $(\mathrm{a}, \mathrm{c})$ and cross-polar $(\mathrm{b}, \mathrm{d})$ radiation patterns at 11.95 $\mathrm{GHz}$ for Y-polarization obtained from measurements $(\mathrm{a}, \mathrm{b})$ and simulations (c, d).

$\mathrm{GHz}$ are shown in Fig. 12 for Y-polarization. This figure shows a qualitative good agreement in both co- and crosspolar components in practically the whole visible range. The measured patterns show a very slight discontinuity at $\mathrm{u}=0$ $\left(\varphi=90^{\circ}\right)$, because the measurements are not identical at the planes $\varphi=90^{\circ}$ and $\varphi=-90^{\circ}$ (edges of the measured angular range), but the differences are within the measurement tolerances. Similar patterns (not shown here) are obtained for the two polarizations at the other frequencies for Tx and Rx. The cross-polar patterns are obtained according to Ludwig's third definition [43]. A quantitative comparison is made by comparing measured and simulated radiation patterns in the principal planes for different frequencies, as shown in the following figures. Figs. 13 and 14 show the radiation patterns for both polarizations at $11.3 \mathrm{GHz}$, which is the lowest

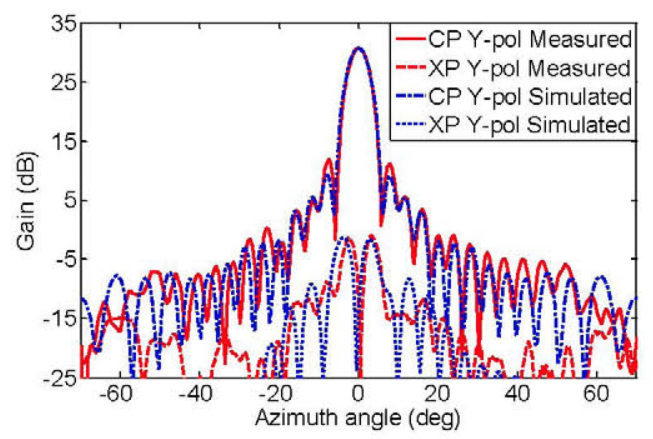

(a)

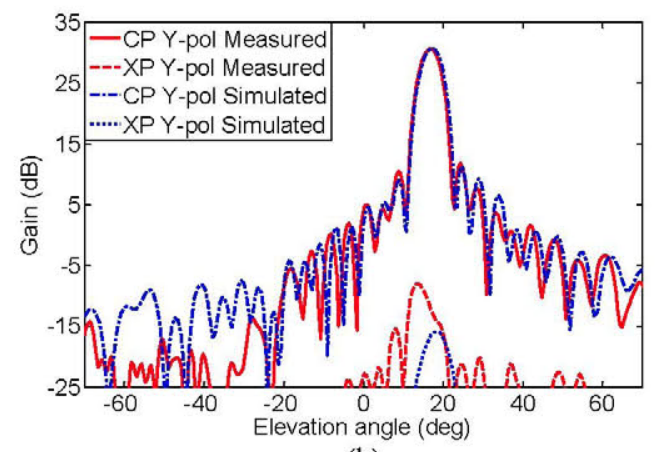

(b)

Fig: 13. Measured and simulated co- and cross-polar radiation patterns at 11.3 $\mathrm{GHz}$ in azimuth (a) and elevation (b) planes for Y-polarization. 
frequency used in the optimizations, while Fig. 15 shows the patterns for Y-polarization at $14.25 \mathrm{GHz}$ (highest frequency in the optimizations). In all the cases, we can observe a very good agreement between simulations and measurements, in both co-polar and cross-polar patterns. The slight discrepancies in the region of side lobes and in the crosspolarization are attributed to small errors in the simulations, manufacturing and measurements. In this work, the feed has

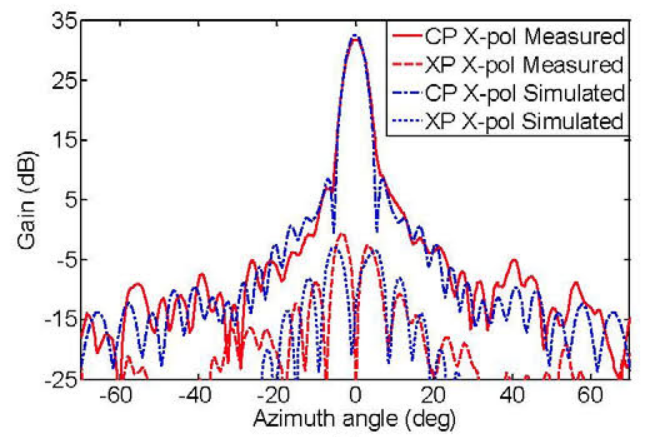

(a)

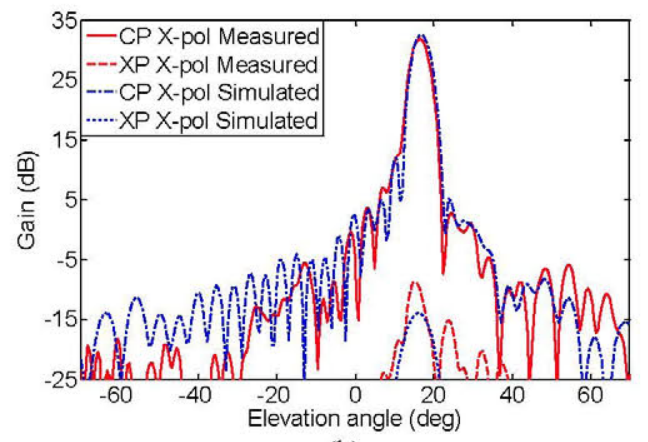

(b)

Fig. 14. Measured and simulated co- and cross-polar radiation patterns at 11.3 GHz in azimuth (a) and elevation (b) planes for X-polarization.

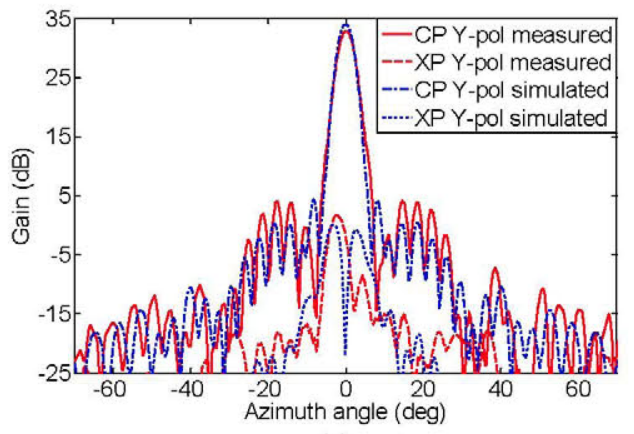

(a)

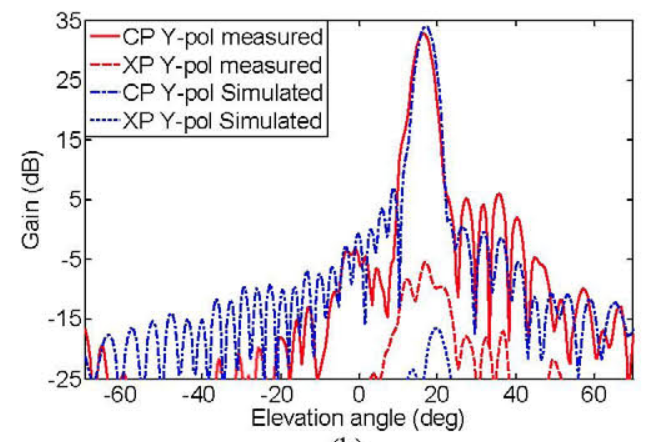

(b)

Fig. 15. Measured and simulated co- and cross-polar radiation patterns at 14.25 GHz in azimuth (a) and elevation (b) planes for Y-polarization. been modeled as an ideal $\cos ^{q}$ function, but the model can be improved by using the near field of the feed [44]. The frame of the reflectarray panel has only been approximately modeled in the simulations by adding 3 rows and 3 columns in the reflectarray edge with a uniform phase-shift.

Note that the azimuth patterns should be symmetric and the cross-polarization in the elevation plane $\left(\mathrm{X}_{\mathrm{R}} Z_{\mathrm{R}}\right)$ should be cancelled because the printed dipoles are symmetric respect to the $X_{R} Z_{R}$ plane. In reality, simulated patterns are symmetric for the co-polar component, but show a slight asymmetry for the cross-polar component. This asymmetry is produced because whereas the cross-polarization of each element is computed by assuming local periodicity, the phasing cells are not symmetric since the dipoles for Y-polarization are shifted by half a period with respect to the dipoles for X-polarization, as shown in Fig. 6(b). This asymmetry of the phasing cell introduced in the numerical simulations produces small asymmetries of the cross-polarization in the azimuth plane and the non-vanishing cross polarization in the elevation plane that appear in the simulated results of Figs. 13 to 15. Additional asymmetries are produced in the prototype during manufacturing and assembling processes, which justifies the small discrepancies between simulated and measured patterns.

Although the antenna has been optimized to maximize the gain at 6 frequencies ( 3 in Tx band and 3 in Rx band), the antenna gain remains stable in the whole range of frequencies from $11.0 \mathrm{GHz}$ to $14.5 \mathrm{GHz}$, as shown in Fig. 16. According to these results, the antenna exhibits a $27 \%$ bandwidth for $2 \mathrm{~dB}$ gain variations in both polarizations. The antenna efficiency is obtained as the ratio between measured gain (in natural units) and the maximum directivity ( $\left.D=4 \pi A /\left(\lambda^{2}\right)\right), A$ being the area of the reflectarray. The resulting antenna efficiency varies from $52 \%$ (at $14.25 \mathrm{GHz}$ ) to $77 \%$ (at $11.95 \mathrm{GHz}$ ) in the whole range of frequencies from $11.00 \mathrm{GHz}$ to $14.25 \mathrm{GHz}$ (when computing this efficiency values, the antenna surface covered by dipoles has been assumed to be a circle of diameter $40 \mathrm{~cm}$ ). The maximum level of measured cross-polarization, also shown in Fig. 16, is at least $28 \mathrm{~dB}$ below the co-polar gain in both polarizations.

To compare the capabilities between the reflectarray proposed here and a reflectarray based on elements with three stacked patches, we have optimized a reflectarray antenna made of the cells proposed in [29] with the same dimensions and in the same frequency bands. The simulations of gain and

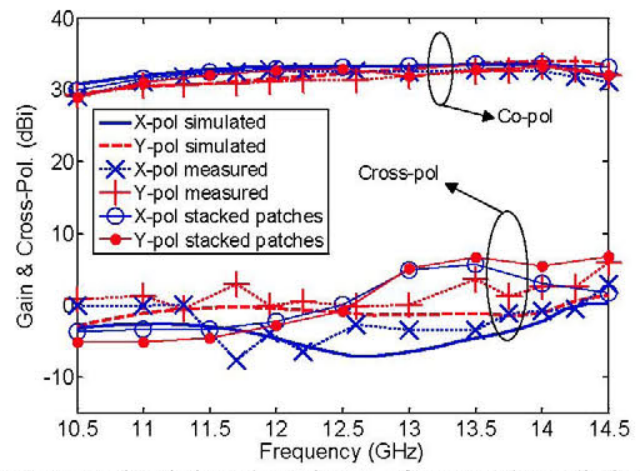

Fig. 16. Antenna gain (dBi) and maximum of cross-polar radiation (dBi) as a function of frequency for the two polarizations. Simulation results for an optimized reflectarray made of cells with 3 stacked patches [29] have also been plotted as a reference. 
maximum of cross-polarization, also included in Fig. 16, show similar values of gain and bandwidth but higher levels of cross-polarization at higher frequencies.

In summary, the results presented in this section demonstrate that the proposed antenna provides high antenna performance in terms of bandwidth, efficiency and low crosspolarization.

\section{DESIGN OF REFLECTARRAY FOR SOUTH AMERICA COVERAGE}

To demonstrate the potential capabilities of the proposed reflectarray element for satellite antennas in Ku-band, a preliminary design has been carried out for a $1.2-\mathrm{m}$ reflectarray antenna to provide the South American coverage defined in [31] at $11.95 \mathrm{GHz}$. The coverage requirements are shown in Fig. 17 and Table I. In this case, the antenna is positioned in the satellite with $Y_{R}$ axis pointing towards East (E), so that $\mathrm{X}$-polarization is vertical (V) and $\mathrm{Y}$-polarization is Horizontal $(\mathrm{H})$. The feed-horn and antenna configuration are the same as those reported in [31]. However, the new periodic cell described in previous section (with four dipoles for each polarization in only two layers and a period of $12 \mathrm{~mm} \times 12 \mathrm{~mm}$ ) is used instead of 3 stacked patches. The reflectarray is elliptical with the elements arranged in 104 rows in $X_{R}$ axis and 99 columns in $Y_{R}$ axis for $X$-polarization, while the number of rows and columns for the phase-shifter cells in Ypolarization are reduced to $103 \times 98$.

The phase distribution required to accomplish the stringent coverage requirements of Table I was obtained for each polarization in [31] by a phase-only pattern synthesis [45][46], assuming a reflectarray made of 6944 periodic cells of $13 \mathrm{mmx} 13 \mathrm{~mm}$ arranged in 96 rows and 92 columns. Since the period for the new reflectarray element is $12 \mathrm{~mm} \times 12 \mathrm{~mm}$, the

TABLE I

GAIN AND CROSS-POL. REQUTREMENTS FOR V-AND H-POLARIZATIONS

\begin{tabular}{ccc}
\hline \hline Zone & Gain $(\mathrm{dBi})$ & $\mathrm{XPD}(\mathrm{dB})$ \\
\hline $\mathrm{SA} 1$ & 28.82 & 31.00 \\
$\mathrm{SA} 2$ & 28.81 & 31.00 \\
$\mathrm{SB}$ & 25.81 & 30.00 \\
$\mathrm{SC} 1$ & 22.81 & 29.00 \\
$\mathrm{SC} 2$ & 20.66 & 27.00 \\
$\mathrm{SD}$ & 19.81 & 27.00 \\
\hline \hline
\end{tabular}

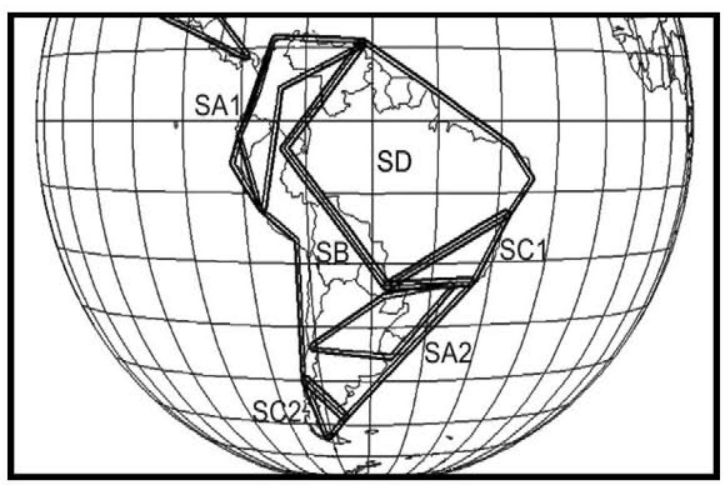

Fig. 17. South American coverage from Amazonas satellite. phase distribution has to be interpolated to the new grid. However, the interpolation in the phase-shift produces some problems because of the steps in $360^{\circ}$. For this reason, an alternative procedure has been used, which consists of the following steps:

1) The radiation patterns are computed by applying the Inverse Discrete Fourier Transform (IDFT) to the field synthesized on the reflectarray surface with period $13-\mathrm{mm} \mathrm{x}$ $13-\mathrm{mm}$. It has been checked that the patterns are compliant with the specified requirements.

2) The complex radiation patterns (magnitude and phase) are used to compute the complex tangential electric field (magnitude and phase) on the reflectarray surface for the new grid with period $12-\mathrm{mm} \times 12-\mathrm{mm}$ by applying DFT. It has been checked that the magnitude of the electric field on the reflectarray is only slightly different (less than $1 \mathrm{~dB}$ in $90 \%$ of the reflectarray elements) from the magnitude of the incident field imposed by the feed-horn. This fact justifies the use of the magnitude of the incident field.

3) The required phase distribution for each polarization is obtained by subtracting the phase of the incident electric field from the phase of the electric field on each reflectarray cell for the $12-\mathrm{mm}$ period, computed in previous step. The phase shift distribution obtained for V-polarization is shown in Fig. 18. A similar phase distribution has been obtained for $\mathrm{H}$-polarization but shifted around $180^{\circ}$ with respect to V-polarization. This phase difference between the two orthogonal polarizations ensures that the lengths of the dipoles controlling the Vpolarization are different as compared to those for $\mathrm{H}$ polarization, and provides more room for the orthogonal dipoles printed on the same layer.

Once the phase distributions on the reflectarray are obtained at $11.95 \mathrm{GHz}$, the dipole lengths are adjusted at each element to match the required phase-shift in both polarizations, by calling the analysis routine as described in previous section. The computed co-polar radiation patterns are represented in the reflectarray coordinate system (with the $\mathrm{Y}_{\mathrm{R}}$ pointing East), so that the $\mathrm{u}(\mathrm{u}=\sin \theta \cos \varphi)$ coordinate is vertical, while $\mathrm{v}$ $(\mathrm{v}=\sin \theta \sin \varphi)$ is horizontal, as shown in Fig. 19. The co-

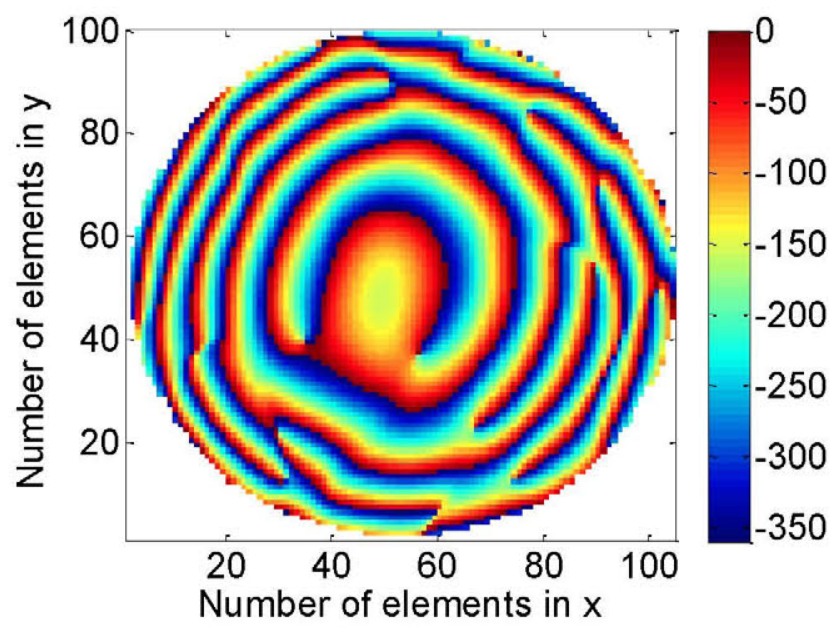

Fig. 18. Required phase shift obtained to generate the South American coverage at $11.95 \mathrm{GHz}$ for $\mathrm{X}$-polarization. 


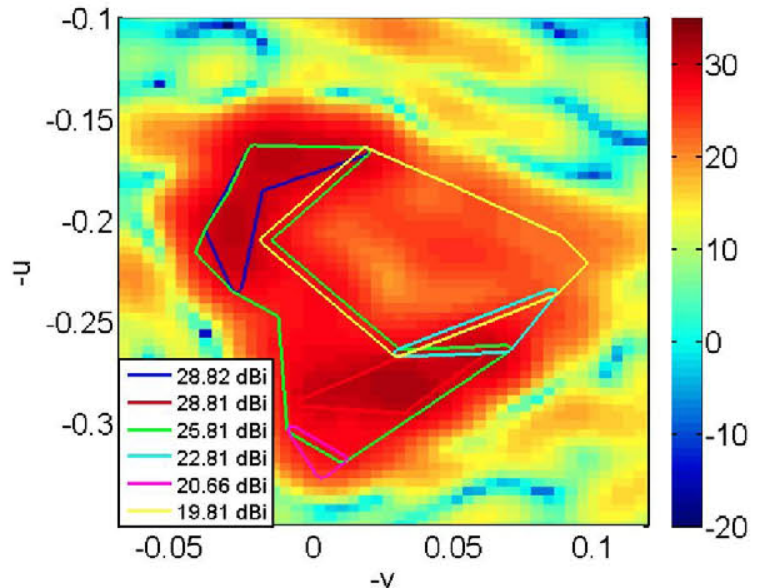

(a)

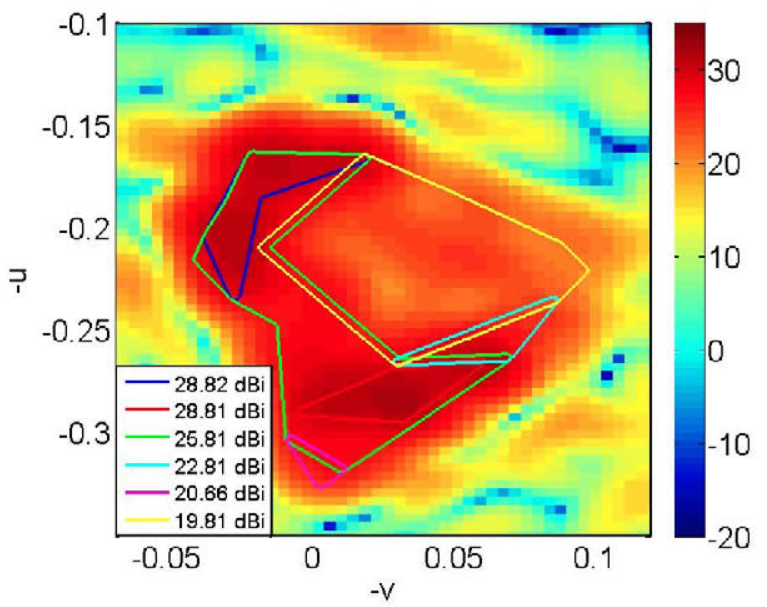

(b)

Fig. 19. Co-polar radiation pattern at $11.95 \mathrm{GHz}$ for H-polarization (a) and V-Polarization (b).

polar radiation pattern fulfills the nominal requirement given in Table I for both polarizations at $11.95 \mathrm{GHz}$. The cross-polar discrimination is represented in Fig. 20. The XPD requirements for $\mathrm{V}$-polarization are fulfilled with a maximum of $60 \mathrm{~dB}$ and minimum of $27 \mathrm{~dB}$ in the SD region. The XPD for $\mathrm{H}$-polarization almost fulfills the requirements with a maximum of $56 \mathrm{~dB}$ and a minimum of $25 \mathrm{~dB}$ in the SD region, where the requirement is $27 \mathrm{~dB}$.

The results from this preliminary design are highly encouraging, although intensive optimizations have to be made for the detailed design in order to ensure the coverage requirements at several frequencies in $\mathrm{Tx}$ and $\mathrm{Rx}$ bands, and to improve the performance in cross-polarization and co-polar isolation.

\section{CONCLUSION}

A broadband dual-polarization reflectarray antenna with improved cross-polarization properties has been proposed in this paper. The reflectarray element provides three degrees of freedom for each polarization, three dipole lengths that can be properly optimized to design spaceborne antennas in Ku-band with low cross-polarization for transmit and receive operation. The flexibility provided by the independent adjustment of the phase for each polarization makes this reflectarray element

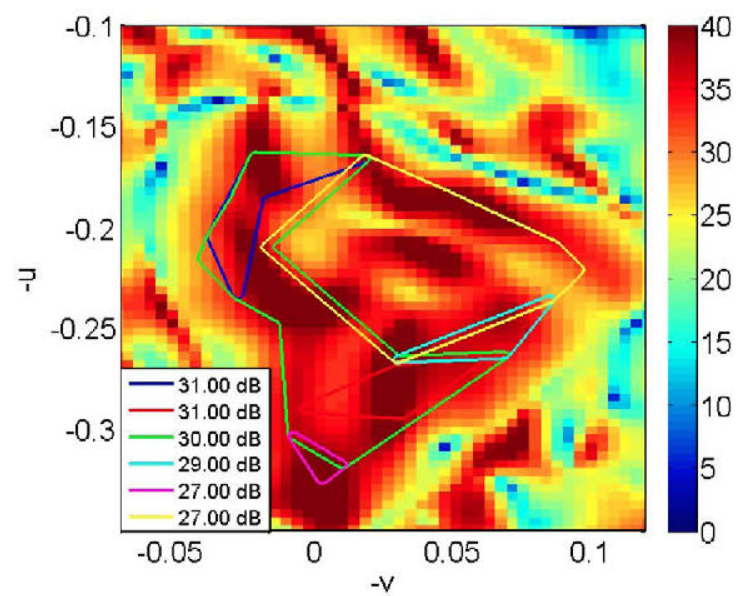

(a)

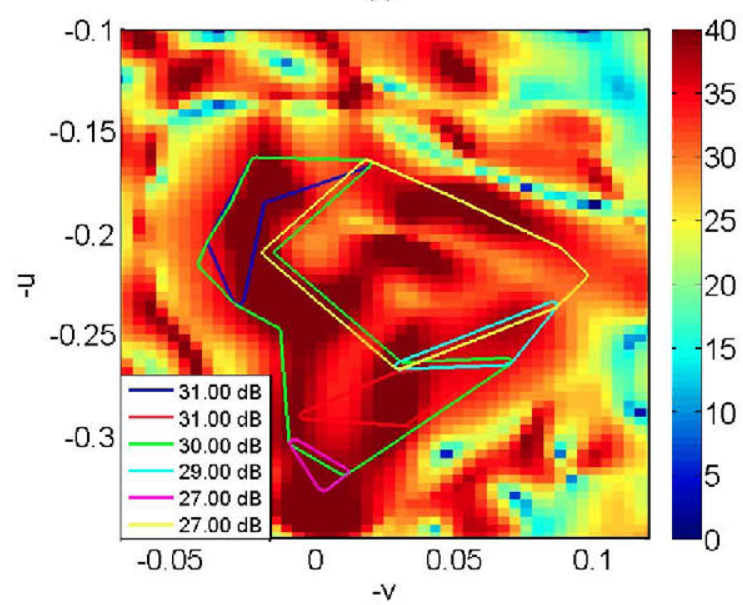

(b)

Fig. 20. Cross-polar discrimination of the radiation pattern at $11.95 \mathrm{GHz}$ for H-polarization (a) and V-Polarization (b).

suitable for the generation of different beams in each polarization, as required in some specific applications.

A $40-\mathrm{cm}$ reflectarray prototype has been optimized to produce a focused beam in a range of frequencies that covers Tx and Rx bands in Ku-band for Fixed Services Satellites. The prototype has been manufactured and tested. The experimental results show a $27 \%$ bandwidth for $2 \mathrm{~dB}$ gain variations, sidelobe levels $20 \mathrm{~dB}$ below the maximum of radiation, and crosspolar radiation at least $28 \mathrm{~dB}$ below the copolar maximum.

A preliminary design of a $1.2 \mathrm{-m}$ antenna for South American coverage has been presented. The simulation results show that the coverage and XPD requirements are practically fulfilled at $11.95 \mathrm{GHz}$, although an additional effort has to be made to design an antenna fully compliant with the requirements in the $\mathrm{Tx}$ and $\mathrm{Rx}$ bands.

The results presented in this paper for both the $40-\mathrm{cm}$ prototype and the $1.2 \mathrm{-m}$ contoured beam antenna show that the proposed reflectarray element can be suitable to design spaceborne antennas in Ku-band that operate in dualpolarization and transmit-receive frequencies.

\section{REFERENCES}

[1] A. Imbriale, S. Gao, L. Boccia, Space Antenna Handbook", John Wiley \& Sons, 2012

[2] J. Huang and J. A. Encinar, Reflectarray antennas. Piscataway, NJ/New York: IEEE Press/Wiley, 2008. 
[3] R. E. Munson and H. Haddad, "Microstrip reflectarray for satellite communication and RCS enhancement and reduction," U.S. patent 4,684,952, Washington, D.C., August 1987.

[4] M. E. Bialkowski, H. J. Song, "Dual linearly polarized reflectarray using aperture coupled microstrip patches," in Proc. 2001 IEEE Antennas and Propag. Society Intl. Symp., vol.3, Boston, MA, USA, pp. 486-489.

[5] E. Carrasco, M. Barba and J. A. Encinar, "Reflectarray element based on aperture-coupled patches with slots and lines of variable length", IEEE Trans. Antennas Propagat, vol.55, no.3, pp.820-825, March 2007.

[6] E. Carrasco, M. Arrebola, J.A. Encinar, M. Barba, "Demonstration of a Shaped Beam Reflectarray Using Aperture-Coupled Delay Lines for LMDS Central Station Antenna," IEEE Trans. Antennas Propagat, vol.56, no. 10 , pp.3103-3111, Oct. 2008.

[7] D.M. Pozar, S.D. Targonski, and H.D. Syrigos, "Design of millimeter wave microstrip reflectarray," IEEE Trans. Antennas Propagat., vol. 45, No. 2, pp. 287-296, February 1997.

[8] P. De Vita, A. Freni, G.L. Dassano, P. Pirinoli, R.E. Zich, "Broadband element for high-gain single-layer printed reflectarray antenna," Electronics Letters, vol.43, no.23, pp. 1247-1249, 2007.

[9] M. R. Chaharmir, J. Shaker, and H. Legay, "Broadband design of a single layer large reflectarray using multi cross loop elements", IEEE Trans. On Antennas and Propag., Vol. 57, no. 10, pp. 3363-3366, October 2009.

[10] R. Florencio, R.R. Boix, E. Carrasco, J.A. Encinar, M. Barba, G. PérezPalomino, 'Broadband reflectarrays made of cells with three coplanar parallel dipoles', Microw. Opt. Technol. Lett., Vol. 56, No. 3, pp. 748 753. Mach 2014.

[11] L. Moustafa, R. Gillard, F. Peris, R. Loison, H. Legay, and E. Girard, "The Phoenix cell: a new reflectarray cell with large bandwidth and rebirth capabilities," IEEE Antennas Wireless Propagat. Lett., Vol. 10, pp. 71-74, 2011.

[12] J. A. Encinar, "Design of two-layer printed reflectarrays using patches of variable size," IEEE Trans. Antennas Propagat., vol. 49, pp. 1403 1410, Oct. 2001.

[13] N. Misran, R. Cahill, V. Fusco, "Design optimisation of ring elements for broadband reflectarray antennas", IEE Proceedings on Microwaves, Antennas and Propagation, Vol. 150, Issue 6, pp. 440-444, Dec. 2003.

[14] H. Legay, B. Salome, E. Labiole, M.A. Milon, D. Cadoret, R. Gillard, R. Chaharmir, J. Shaker, "Reflectarrays for satellite telecommunication antennas," in Proc. $2^{\text {nd }}$ European Conf. on Antennas and Propagation, EuCAP 2007, Edinburgh, UK, pp. 1-6.

[15] D. M. Pozar, S. D. Targonski, and R. Pokuls, "A shaped-beam microstrip patch reflectarray," IEEE Trans. Antennas Propagat., vol. 47, no. 7, pp. 1167-1173, July 1999 .

[16] J. A. Encinar, L. Datashvili, J. A. Zornoza, M. Arrebola, M. SierraCastañer, J. L. Besada, H. Baier, and H. Legay, "Dual-polarization dualcoverage reflectarray for space applications," IEEE Trans. on Antennas and Propag., Vol. 54, no. 10, pp. 2828-2837, Oct. 2006.

[17] S. R. Rengarajan, "Reflectarrays of rectangular microstrip patches for dual-polarization dual-beam radar interferometers", Progress In Electromagnetics Research, Vol. 133, pp. 1-15, 2013.

[18] H. Legay, Y. Cailloce, O. Vendier, G. Caille, J. Perruisseau-Carrier, M. Lathi, J.P. Polizzi, U. Oestermann, P. Pons, N. Raveu, "Satellite antennas based on MEMS tunable reflectarrays," in Proc. $2^{\text {nd }}$ European Conf. on Antennas and Propagation, 2007, Edinburgh, UK, pp. 1-6.

[19] E. Carrasco, M. Barba, B. Reig, C. Dieppedale, J.A. Encinar, "Characterization of a reflectarray gathered element with electronic control using ohmic RF MEMS and patches aperture-coupled to a delay line," IEEE Trans. Antennas and Propagat. , vol. 60, no.9, pp.41904201, Sept. 2012.

[20] D. M. Pozar, "Bandwidth of reflectarrays", Electr. Lett. vol. 39, no. 21, pp. 1490-1491, Oct. 2003.

[21] R. Florencio, R.R Boix, J.A. Encinar, "Comparative study of reflectarrays based on cells with three coplanar dipoles and reflectarrays based on cells with three stacked patches," in Proc. 6th Edition EUCAP, Prague, Czech Republic, March 2012.

[22] J. Huang, "Bandwidth study of microstrip reflectarray and a novel phased reflectarray concept", in Proc. IEEE Antennas and Propagation Society Intl. Symp., 1995, Newport Beach, CA, USA, pp. 582-585.

[23] Jose A. Encinar and J. Agustin Zornoza, "Three-layer printed reflectarrays for contoured beam space applications," IEEE Trans. Antennas Propagat., vol. 52, no. 5, pp. 1138-1148, May 2004.

[24] E. Carrasco, J. A. Encinar and M. Barba, "Bandwidth improvement in large reflectarrays by using true-time-delay," IEEE Trans. Antennas Propagat., Vol. 56, pp. 2496-2503, Aug. 2008.
[25] A. Roederer, "Reflector antenna comprising a plurality of panels", U.S. patent 6411255 , June 2002.

[26] H. Legay, D. Bresciani, E. Labiole, R. Chiniard, E. Girard, G. Caille, D. Calas, R. Gillard, R. Toso, "A $1.3 \mathrm{~m}$ facetted reflectarray in Ku band," in Proc. 15th Intl. Symp. Antenna Technology and Applied Electromagnetics (ANTEM), Toulouse, France, 2012, pp. 1-4.

[27] M. E. Cooley, T. J. Chwalek, "Method for improving pattern bandwidth of shaped beam reflectarrays", U.S. patent $006031506,2000$.

[28] C. Tienda, M. Arrebola, J.A. Encinar, G. Toso, "Analysis of parabolic reflectarray in dual-reflector configuration," in Proc. of the $4^{\text {th }}$ European Conf. on Antennas and Propagation (EuCAP), Barcelona, Spain, 2010.

[29] J. A. Encinar, J. A. Zornoza, "Broadband design of three-layer printed reflectarrays," IEEE Trans. on Antennas and Propag., Vol. 51, no. 7, pp. 1662-1664, July 2003.

[30] J. A. Encinar, M. Arrebola, M. Dejus and C. Jouve, "Design of a 1metre reflectarray for DBS application with $15 \%$ bandwidth", in Proc. of the European Conference on Antennas and Propagation (EuCAP 2006), Nice, France, 2006.

[31] J. A. Encinar, M. Arrebola, L. F. de la Fuente, G. Toso, "A TransmitReceive Reflectarray Antenna for Direct Broadcast Satellite Applications" IEEE Trans. on Antennas and Propagat. Vol. 59, no. 9; pp. 3255-3264, Sept. 2011.

[32] M. Zhou, S.B. Sorensen, O.S. Kim, E. Jorgensen, P. Meincke, O. Breinbjerg, "Direct optimization of printed reflectarrays for contoured beam satellite antenna applications," IEEE Trans. on Antennas and Propagat., vol.61, no.4, pp.1995-2004, April 2013.

[33] M. Zhou, S.B. Sorensen, O.S. Kim, E. Jorgensen, P. Meincke, O. Breinbjerg, G. Toso, "The generalized direct optimization technique for printed reflectarrays," IEEE Trans. on Antennas and Propagat., vol.62, no.4, pp.1690-1700, April 2014.

[34] J. R. Profera, E. Charles, "Reflectarray Antenna for Communication Satellite Frequency Re-use Applications", U.S. patent 543809, August 1996.

[35] K. C. Clancy, m. e. Cooley, D. Bressler, "Apparatus and method for reducing polarization cross-coupling in cross dipole reflectarrays", U.S. patent 2001/0050653 A1, March 2000.

[36] J.A. Encinar, A. Pedreira, "Flat reflector antenna in printed technology with improved bandwidth and separate polarizations", Spanish patent P200401382, 2004.

[37] R. Mittra, C. H. Chan, and T. Cwik, "Techniques for analyzing frequency selective surfaces-A review," Proc. IEEE. vol. 76, no. 12, pp. 1593-1615, Dec. 1988

[38] F. Mesa, R. Marqués, and M. Horno, "A general algorithm for computing the bidimensional spectral Green's dyads in multilayered complex bianisotropic media: the equivalent boundary method," IEEE Trans. Microwave Theory Tech, vol. 39, pp. 1640-1649, Sept. 1991.

[39] CST Microwave Studioß, [online: www.cst.com]

[40] G. Perez-Palomino, J. A. Encinar, M. Barba, and E. Carrasco, "Design and evaluation of multi-resonant unit-cells based on liquid crystals for reconfigurable reflectarrays," IET Microw. Antennas Propag., vol. 6, no. 3, pp. 348-354, Feb. 2012.

[41] S.D. Targonski, D.M. Pozar, "Minimization of beam squint in microstrip reflectarrays using an offset feed," in Proc. IEEE Antennas and Propagation Society International Symposium, Baltimore, MD, USA, 1996. vol.2, pp.1326-1329.

[42] M. Zhou, S. B. Sørensen, E. Jørgensen, P. Meincke, O. S. Kim, and O. Breinbjerg, "An accurate technique for calculation of radiation from printed reflectarrays," IEEE Antennas Wireless Propag. Lett., vol. 10, pp. 1081-1084, 2011

[43] A. C. Ludwig, "The definition of cross-polarization," IEEE Trans. Antennas Propagat., vol. 21, pp. 116-119, Jan. 1973.

[44] M. Arrebola, Y. Alvarez, J. A. Encinar, F. Las-Heras, "Accurate analysis of printed reflectarrays considering the near field of the primary feed", IET Microw. Antennas Propag., vol. 3, no. 2, pp. 187-194, March 2009.

[45] O. Bucci, G. Franceschetti, G. Mazzarella, and G. Panariello, "Intersection approach to array pattern synthesis," IEE Proceedings, vol. 137, Pt. H, no.6, pp. 349-357, Dec. 1990.

[46] J. Agustín Zornoza, Jose A. Encinar, "Efficient phase-only synthesis of contoured beam patterns for very large reflectarrays," International Journal of RF and Microwave Computer-Aided Engineering, pp. 415 423, Sept. 2004. 


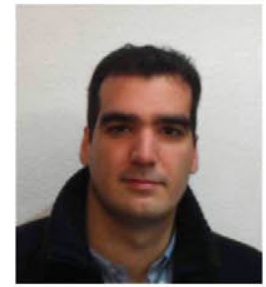

Rafacl Florencio received the Licenciado degree in Physics from the University of Seville, Spain, in 2008, and the "Master in Communications Technology and Systems" from the Technical University of Madrid (UPM), Spain, in 2010.

In 2010 he joined the Electronics and Electromagnetism Department, University of Seville, where he is currently working towards the Ph.D.

Mr. Florencio was the recipient of a Scholarship financed by the Junta de Andalucía.

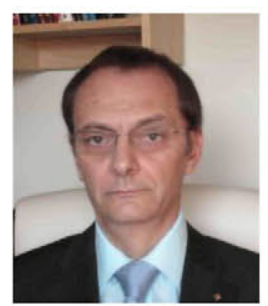

José A. Encinar (S'81-M'86-SM'09-FM'10) was born in Madrid, Spain. He received the Electrical Engineer and $\mathrm{Ph} . \mathrm{D}$. degrees, both from Universidad Politécnica de Madrid (UPM), in 1979 and 1985, respectively.

Since January 1980, he has been with the Applied Electromagnetism Group at UPM, as a Teaching and Research Assistant from 1980 to 1982, as an Assistant Professor from 1983 to 1986, and as Associate Professor from 1986 to 1991. From February to October of 1987 , he was a Postdoctoral Fellow of the NATO Science Program with the Polytechnic University, Brooklyn, NY, USA. Since 1991 he is a Professor of the Electromagnetism and Circuit Theory Group, currently in the Department of Signals, Systems and Radio Communications at UPM. He was a Visiting Professor with the Laboratory of Electromagnetics and Acoustics at Ecole Polytechnique Fédérale de Lausanne (EPFL), Switzerland, in 1996, and with the Institute of Electronics, Communication and Information Technology (ECIT), Queen's University Belfast, U.K., in 2006 and 2011. His research interests include numerical techniques for the analysis of multi-layer periodic structures, design of frequency selective surfaces, printed arrays and reflectarrays.

Prof. Encinar has co-authored more than 150 journal and conference papers, one book and several book chapters. He is holder of five patents on array and reflectarray antennas. He was a co-recipient of the $2005 \mathrm{H}$. A. Wheeler Applications Prize Paper Award and the 2007 S. A. Schelkunoff Transactions Prize Paper Award, given by IEEE Antennas and Propagation Society. He has been a member of the Technical Programme Comity of several International Conferences (European Conference on Antennas and Propagation, ESA Antenna Workshops, Loughborough Antennas \& Propagation Conference).

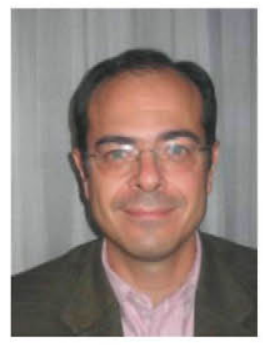

Rafael R. Boix (M'96) received the Licenciado and Doctor degrees in Physics from the University of Seville, Spain, in 1985 and 1990 respectively.

Since 1986, he has been with the Electronics and Electromagnetism Department, University of Seville, where he became Tenured Professor in 2010. His current research interests are focused on the efficient numerical analysis of periodic planar multilayered structures with applications to the design of frequency selective surfaces and reflectarray antennas.

Vicente Losada was born in Sao Paulo (Brazil) in February 1969. He received the Licenciado and $\mathrm{Ph} . \mathrm{D}$. degrees from the University of Sevilla, Seville, Spain, in 1992 and 1997, respectively, both in Physics.

$\mathrm{He}$ has been Associate Professor in the Department of Applied Physics I, University of Seville, since 2004. His research interest include manufacture and near field and far-field measurements techniques for antennas.

He acts as reviewer for IEEE-AP journal.

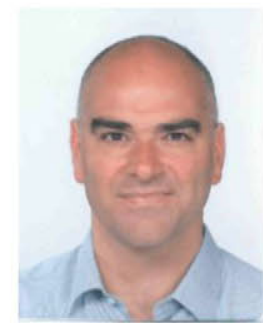

Giovanni Toso (S'93-M'00-SM'07) received the Laurea Degree (summa cum laude) and the Ph.D. in Electrical Engineering from the University of Florence, Florence, Italy, in 1992 and 1995, respectively.

In 1996 he was visiting scientist at the Laboratoire d'Optique Electromagnétique, University of Aix-Marseille III, Marseille, France. From 1997 to 1999 he was a Post Doctoral student at the University of Florence. In 1999 he was a visiting scientist at the University of California, Los Angeles (UCLA). In the same year he received a scholarship from Thales Alenia Space (Rome, Italy) and he has been appointed researcher in a Radioastronomy Observatory of the Italian National Council of Researches (CNR). Since 2000 he is with the Antenna and Submillimeter Section of the European Space and Technology Centre of the European Space Agency, ESA ESTEC, Noordwijk, The Netherlands. He has been initiating and contributing to several $R \& D$ activities on satellite antennas based on arrays, reflectarrays, constrained lenses and reflectors.

G. Toso has co-authored more than 50 technical papers published in peer reviewed professional journals, more than 200 papers published in international conferences' proceedings, and more than 10 international patents. In 2009 he has been coeditor of the Special Issue on Active Antennas for Satellite Applications in the International Journal of Antennas and Propagation. In April 2014 he has been co-guest editor, with Dr. R. Mailloux, of the Special Issue on Innovative Phased Array Antennas based on NonRegular Lattices and Overlapped Subarrays published in the IEEE Transactions on Antennas and Propagation. G. Toso is an Associate Editor of the IEEE Transactions on Antennas and Propagation. 